\title{
Exploring the hyperchargeless Higgs triplet model up to the Planck scale
}

\author{
Najimuddin Khan ${ }^{\mathrm{a}}$ \\ Discipline of Physics, Indian Institute of Technology Indore, Khandwa Road, Simrol, Indore 453 552, India
}

Received: 17 April 2017 / Accepted: 26 March 2018 / Published online: 26 April 2018

(C) The Author(s) 2018

\begin{abstract}
We examine an extension of the SM Higgs sector by a Higgs triplet taking into consideration the discovery of a Higgs-like particle at the LHC with mass around $125 \mathrm{GeV}$. We evaluate the bounds on the scalar potential through the unitarity of the scattering matrix. Considering the cases with and without $\mathbb{Z}_{2}$-symmetry of the extra triplet, we derive constraints on the parameter space. We identify the region of the parameter space that corresponds to the stability and metastability of the electroweak vacuum. We also show that at large field values the scalar potential of this model is suitable to explain inflation.
\end{abstract}

\section{Introduction}

The revelation of the Higgs boson [1-3] in 2012 at the Large Hadron Collider (LHC) confirmed the existence of all the Standard Model (SM) particles and showed the Higgs mechanism to be responsible for electroweak symmetry breaking (EWSB). So far, the LHC, operated with $p p$ collision energy at $\sqrt{s} \sim 8$ and $13 \mathrm{TeV}$, has not found any signature of new physics beyond the standard model (BSM). However, various theoretical issues, such as the hierarchy problem related to the mass of the Higgs, mass hierarchy and mixing patterns in the leptonic and quark sectors, suggest the need for new physics beyond the SM. Different experimental observations, such as the non-zero neutrino mass, the baryon-antibaryon asymmetry in the Universe, the mysterious nature of dark matter (DM) and dark energy, and inflation in the early Universe indicate the existence of new physics. Moreover, the measured properties of the Higgs boson with mass $\sim 125$ $\mathrm{GeV}$ are consistent with those of the scalar doublet as predicted by the SM. However, the experimental data [4] still comfortably allow for an extended scalar sector, which may also be responsible for the EWSB.

a e-mail: phd11125102@iiti.ac.in; khanphysics.123@gmail.com
The present experimental values of the SM parameter of the Lagrangian indicate that if the validity of the $\mathrm{SM}$ is extended up to the Planck mass $\left(M_{\mathrm{Pl}}=1.2 \times 10^{19} \mathrm{GeV}\right)$, a second, deeper minimum is located near the Planck mass such that the EW vacuum is metastable. The transition lifetime of the $\mathrm{EW}$ vacuum to the deeper minimum is finite $\tau_{\mathrm{EW}} \sim 10^{300}$ years [5-16]. The EW vacuum remains metastable even after adding extra scalar particles to the SM, which has been discussed in Refs. [15-19].

In this work, we add a real hypercharge $Y=0$ scalar triplet to the SM. In the literature, this model is termed the hyperchargeless Higgs triplet model, HTM $(Y=0)$ [20]. We consider both the neutral $C P$-even component of the SM doublet and the extra scalar triplet take part in the EWSB. Including radiative corrections, we check the validity of the parameters of the model up to the Planck mass $M_{\mathrm{Pl}}$. We review various theoretical and experimental bounds of this model. In this work, we especially discuss the unitary bounds of the quartic couplings of the scalar potential. To the best of our knowledge, the unitary bounds of this model were not discussed in the literature. Next, we impose a $\mathbb{Z}_{2}$-symmetry such that an odd number of scalar particles of the triplet do not couple with the SM particles. The lightest neutral scalar particle does not decay and becomes stable. This scalar field can be taken as a viable DM candidate which may fulfill the relic abundance of the Universe. In this context, it is instructive to explore whether these extra scalars can also prolong the lifetime of the Universe. In this model, we find new regions in the parameter space of this model in which the EW vacuum remains metastable. We also consider that the extra neutral scalar field (also compatible as a viable dark matter candidate) can act as an inflaton. We show that this scalar field is able to explain the inflationary observables.

A detailed study of the HTM $(Y=0)$ parameter space, which is valid up to $1 \mathrm{TeV}$, has been performed in Refs. [21]. Two different renormalization schemes, electroweak precision, and decoupling of Higgs triplet scenario have been 
discussed in Ref. [22]. Using the electroweak precision test (EWPT) data and a one-loop correction to the $\rho$ parameter, the Higgs mass range has been predicted in Refs. [23-27]. The detailed structure of the vacuum of the scalar potential at tree level has been studied in Ref. [28]. The constraints on the parameter spaces from the recent LHC $\mu_{\gamma \gamma}$ and $\mu_{Z \gamma}$ data have been discussed in Ref. [29]. The LHC and future collider experiments with high luminosity can be used as an useful tool to detect these extra scalar particles through vector bosons scatterings [30]. More recently, the inert scalar triplet has been investigated in the context of dark matter direct and indirect detection [31-33]. The heavier inert fields can decay through one loop via extra Majorana fermions [34,35]. This model has the required ingredients to realize a successful leptogenesis which can explain the matter asymmetry in the Universe [34,35]. Multi-component dark matter has been investigated $[36,37]$ in HTM with extra scalar multiplets of the $S U(2)$ representation.

The paper is organized as follows. Section 2 starts with a detailed description of the HTM $(Y=0)$ model. We discuss detailed constraints in Sect. 3. Considering the lightest $\mathbb{Z}_{2}$-odd neutral particle as a viable DM, we analyze the scalar potential up to the Planck mass and identify regions of parameter space corresponding to the stable and metastable EW vacuum in Sect. 4. We explain inflation as well in Sect. 5. Finally we conclude in Sect. 6.

\section{Model}

We consider a model with a real Higgs doublet, $\Phi$, and a real, isospin $I=1$, hypercharge $Y=0$ triplet $T$. The extra scalar triplet consists of a pair of singly charged fields and a $C P$-even neutral scalar field. The doublet and triplet scalar are conventionally written as [22]

$\Phi=\left(\begin{array}{c}G_{1}^{+} \\ \frac{1}{\sqrt{2}}\left(v_{1}+h^{0}+i G^{0}\right)\end{array}\right), \quad T=\left(\begin{array}{c}\eta^{+} \\ v_{2}+\eta^{0} \\ -\eta^{-}\end{array}\right)$.

The kinetic part of the Lagrangian is given by

$\mathcal{L}_{k}=\left|D_{\mu} \Phi\right|^{2}+\frac{1}{2}\left|D_{\mu} T\right|^{2}$

where the covariant derivatives are defined by

$$
\begin{aligned}
& D_{\mu} \Phi=\left(\partial_{\mu}+i \frac{g_{2}}{2} \sigma^{a} W^{a}+i \frac{g_{1}}{2} Y B_{\mu}\right) \Phi \text { and } \\
& D_{\mu} T=\left(\partial_{\mu}+i g_{2} t_{a} W^{a}\right) T,
\end{aligned}
$$

where $W_{\mu}^{a}(a=1,2,3)$ are the $S U(2)_{L}$ gauge bosons, corresponding to three generators of $S U(2)_{L}$ group and $B_{\mu}$ is the $U(1)_{Y}$ gauge boson. The $\sigma^{a}(a=1,2,3)$ are the Pauli matrices, and $t_{a}$ can be written as follows:

$$
\begin{aligned}
t_{1} & =\frac{1}{\sqrt{2}}\left(\begin{array}{ccc}
0 & 1 & 0 \\
1 & 0 & 1 \\
0 & 1 & 0
\end{array}\right), \quad t_{2}=\frac{1}{\sqrt{2}}\left(\begin{array}{ccc}
0 & -i & 0 \\
i & 0 & -i \\
0 & i & 0
\end{array}\right), \\
t_{3} & =\left(\begin{array}{ccc}
1 & 0 & 0 \\
0 & 0 & 0 \\
0 & 0 & -1
\end{array}\right) .
\end{aligned}
$$

The scalar potential is such that both the neutral $C P$-even component of the SM doublet and the extra scalar triplet receive vacuum expectation values (VEVs) and thus take part in the EWSB. After EWSB, one of the linear combinations of charged scalar fields of the scalar doublet and the triplet is eaten by the $W$ boson, which becomes massive, and other orthogonal combinations of these fields become massive charged scalar fields. Similarly, a pseudoscalar of the scalar doublet becomes the longitudinal part of the massive $Z$ gauge boson. This scalar may give rise to a signature through the scattering of vector bosons [30] in collider experiments. The spontaneous EWSB generates masses for the $W$ and $Z$ bosons, thus:

$M_{W}^{2}=\frac{g_{2}^{2}}{4}\left(v_{1}^{2}+4 v_{2}^{2}\right)$, and $M_{Z}^{2}=\frac{g_{2}^{2}}{4 c_{\theta}^{2}} v_{1}^{2}$,

where $c_{W} \equiv \cos \theta_{W}=g_{2} / \sqrt{g_{1}^{2}+g_{2}^{2}}$ and $s_{W} \equiv \sin \theta_{W}$. The scalar doublet VEV $v_{1}$ and the triplet VEV $v_{2}$ are related to the SM VEV by $v_{\mathrm{SM}}(\equiv 246.221 \mathrm{GeV})=\sqrt{v_{1}^{2}+4 v_{2}^{2}}$.

One can see that this model violates custodial symmetry at tree level,

$\rho=\frac{M_{W}^{2}}{M_{Z}^{2} c_{W}^{2}}=1+4 \frac{v_{2}^{2}}{v_{1}^{2}}$.

The experimental value of $\rho$ is $1.0004 \pm 0.00024$ [38] at $1 \sigma$. Hence, $\delta \rho \approx 0.0004 \pm 0.00024$ and we will adopt the bound $\delta \rho \leq 0.001$. This puts stringent constraints on $v_{2}$ and we find that $v_{2}$ should be less than $4 \mathrm{GeV}$.

The tree-level scalar potential with the Higgs doublet and the real scalar triplet is invariant under $S U(2)_{L} \times U(1)_{Y}$ transformation. This is given by

$$
\begin{aligned}
V(\Phi, T)= & \mu_{1}^{2}|\Phi|^{2}+\frac{\mu_{2}^{2}}{2}|T|^{2}+\lambda_{1}|\Phi|^{4}+\frac{\lambda_{2}}{4}|T|^{4} \\
& +\frac{\lambda_{3}}{2}|\Phi|^{2}|T|^{2}+\lambda_{4} \Phi^{\dagger} \sigma^{a} \Phi T_{a} .
\end{aligned}
$$

We have the following minimization conditions of the treelevel scalar potential:

$\mu_{1}^{2}=\frac{1}{2}\left\{2 \lambda_{4} v_{2}-\left(2 \lambda_{1} v_{1}^{2}+\lambda_{3} v_{2}^{2}\right)\right\}$,
$\mu_{2}^{2}=\frac{1}{2 v_{2}}\left\{\lambda_{4} v_{1}^{2}-\lambda_{3} v_{1}^{2} v_{2}-2 \lambda_{2} v_{2}^{3}\right\}$. 
After electroweak symmetry breaking, the squared mass matrix can be expressed as $6 \times 6$ for the scalar fields $\left(G_{1}^{ \pm}, \eta^{ \pm}, \eta^{0}\right.$ and $\left.h^{0}\right)$. This matrix is composed of three $2 \times 2$ submatrices with bases, $\left(G_{1}^{+}, \eta^{+}\right),\left(G_{1}^{-}, \eta^{-}\right)$and $\left(h^{0}, \eta^{0}\right)$. After rotating these fields into the mass basis, we get four physical mass eigenstates $\left(H^{ \pm}, h, H\right)$. The remaining two states $\left(G^{ \pm}\right)$and $G^{0}$ become massless Goldstone bosons.

The physical masses of the particles are given by

$M_{h}^{2}=\frac{1}{2}\left[(B+A)-\sqrt{(B-A)^{2}+4 C^{2}}\right]$,

$M_{H}^{2}=\frac{1}{2}\left[(B+A)+\sqrt{(B-A)^{2}+4 C^{2}}\right]$,

$M_{H^{ \pm}}^{2}=\lambda_{4} \frac{\left(v_{1}^{2}+4 v_{2}^{2}\right)}{2 v_{2}}$,

where

$A=2 \lambda_{1} v_{1}^{2}, \quad B=\frac{\lambda_{4} v_{1}^{2}+4 \lambda_{2} v_{2}^{3}}{2 v_{2}}, \quad$ and

$C=-\lambda_{4} v_{1}+\lambda_{3} v_{1} v_{2}$.

The mixing between the doublet and triplet in the charged and $C P$-even scalar sectors are, respectively, given by

$$
\begin{aligned}
& \left(\begin{array}{l}
h \\
H
\end{array}\right)=\left(\begin{array}{cc}
c_{\gamma} & s_{\gamma} \\
-s_{\gamma} & c_{\gamma}
\end{array}\right)\left(\begin{array}{c}
h^{0} \\
\eta^{0}
\end{array}\right), \\
& \left(\begin{array}{l}
G^{ \pm} \\
H^{ \pm}
\end{array}\right)=\left(\begin{array}{cc}
c_{\beta} & s_{\beta} \\
-s_{\beta} & c_{\beta}
\end{array}\right)\left(\begin{array}{c}
G_{1}^{ \pm} \\
\eta^{ \pm}
\end{array}\right),
\end{aligned}
$$

where

$$
\begin{aligned}
s_{\gamma}(\equiv \sin \gamma) & =\sqrt{\frac{\sqrt{(B-A)^{2}+4 C^{2}}-(B-A)}{2 \sqrt{(B-A)^{2}+4 C^{2}}}} \text { and } \\
\tan \beta & =\frac{2 v_{2}}{v_{1}} .
\end{aligned}
$$

In the large $\mu_{2}^{2}$ and small $v_{2}$ limits, one can express $\sin \gamma$ and $\sin \beta$ as

$$
\begin{aligned}
& s_{\gamma}=\sqrt{\frac{1}{2}-\frac{1}{2 \sqrt{1+16 \frac{v_{2}^{2}}{v_{1}^{2}}}}} \approx 0 \text { and } \\
& s_{\beta}=\frac{2 v_{2}}{\sqrt{v_{1}^{2}+4 v_{2}^{2}}} \approx 0 .
\end{aligned}
$$

In these limits, the quartic $\lambda_{1,2,3}$ and $\lambda_{4}$ can be written as

$$
\begin{aligned}
& \lambda_{1}=\frac{M_{h}^{2}}{2 v_{1}^{2}}, \quad \lambda_{2}=\frac{2\left(M_{H}^{2}-M_{H^{ \pm}}^{2}\right)}{v_{1}^{2} s_{\beta}^{2}}, \\
& \lambda_{3}=\frac{2\left(M_{H^{ \pm}}^{2}-\left(s_{\gamma} / s_{\beta}\right) M_{H}^{2}\right)}{v_{1}^{2}}, \quad \lambda_{4}=\frac{s_{\beta} M_{H^{ \pm}}^{2}}{v_{1}} .
\end{aligned}
$$

In the same limits, if $M_{H^{ \pm}}$and $M_{H}$ are very heavy compared with $M_{h}$, then $M_{H^{ \pm}}$and $M_{H}$ become degenerate (see Eqs. (2.9) and (2.10)). If the mass difference between $M_{H^{ \pm}}$ and $M_{H}$ is large, then the quartic couplings $\lambda_{2,3}$ will violate the perturbativity and unitarity bounds (see Sects. 3.2 and 3.3).

The SM gauge symmetry, $S U(2)_{L}$, prohibits direct coupling of the SM fermions with the scalar fields of the triplet. The couplings of the new scalar fields $\left(H, H^{ \pm}\right)$with SM fermions are generated after the EWSB. The strengths of $H \bar{f} f$ (the $f$ are the up, down quarks and charged leptons) are proportional to $\sin \gamma$. The couplings $H^{+} \bar{v}_{l} l^{-}$and $H^{+} \bar{u} d$ are proportional to $\sin \beta$.

\section{Constraints on the hyperchargeless Higgs triplet model}

The parameter space of this model is constrained by theoretical considerations like the absolute vacuum stability, perturbativity, and unitarity of the scattering matrix. In the following, we will discuss these theoretical bounds and the constraints of the Higgs to diphoton signal strength from the LHC and the electroweak precision measurements.

\subsection{Vacuum stability bounds}

A necessary condition for the stability of the vacuum comes from requiring that the scalar potential is bounded from below, i.e., it should not approach negative infinity along any direction of the field space for large field values. For $h^{0}, \eta^{0, \pm} \gg v_{1,2}$, the quadratic terms $\mu_{1}^{2}|\Phi|^{2}, \frac{\mu_{2}^{2}}{2}|T|^{2}$ and $\lambda_{4} \Phi^{\dagger} \sigma^{a} \Phi T_{a}$ of the scalar potential in Eq. (2.6) are negligibly small compared with the other quartic terms, so the scalar potential is given by

$$
\begin{aligned}
V\left(h^{0}, \eta^{0}, \eta^{ \pm}\right)=\frac{1}{4} & {\left[\lambda_{1}\left(h^{0}\right)^{4}+\lambda_{2}\left(\eta^{2}+2 \eta^{+} \eta^{-}\right)^{2}\right.} \\
& \left.+\lambda_{3}\left(h^{0}\right)^{2}\left(\eta^{2}+2 \eta^{+} \eta^{-}\right)\right] .
\end{aligned}
$$

The potential can be written in a symmetric matrix with basis $\left\{\left(h^{0}\right)^{2},\left(\eta^{0}\right)^{2}, \eta^{-} \eta^{+}\right\}$. Using the copositivity criteria [104], one can calculate the required conditions for the absolute stability/bounded from below of the scalar potential. The treelevel scalar potential $V(\Phi, T) \equiv V\left(h^{0}, \eta^{0}, \eta^{ \pm}\right)$is absolutely stable if

$\lambda_{1}(\Lambda) \geq 0, \quad \lambda_{2}(\Lambda) \geq 0, \quad \lambda_{3}(\Lambda) \geq-2 \sqrt{\lambda_{1}(\Lambda) \lambda_{2}(\Lambda)}$.

The coupling constants are evaluated at a scale $\Lambda$ using RGEs. In this study, we use the SM RGEs up to three loops which have been given in Refs. [52-55]. The triplet contributions are taken up to two loops which are presented in Appendix A. If the quantum corrections are included to the scalar potential, then there is a possibility to form a minimum along the Higgs field direction near the Planck mass $M_{\mathrm{Pl}}$. For 
negative $\lambda_{1}(\Lambda)$ the minimum at the energy scale $\Lambda$ becomes deeper than the EW minimum and vice versa. In these situations, the above conditions in Eq. (3.2) become more complicated. These modifications will be shown in Sect. 4.2. As $\lambda_{3}$ gives a positive contribution to the running of $\lambda_{2}, \lambda_{2}$ remains positive up to the Planck mass $M_{\mathrm{P} 1}$. Hence, it is clear that no extra minimum will develop along the new scalar field directions. The sign and the value of $\lambda_{3}$ can change the Higgs diphoton signal strength and the stability of the EW vacuum. The importance of the sign of $\lambda_{3}$ will be discussed in Sects. 3.5 and 4.3 .

\subsection{Perturbativity bounds}

To ensure that the radiatively improved scalar potential $V(\Phi, T)$ remains perturbative at any given energy scale $(\Lambda)$, one must impose the following conditions:

$$
\left|\lambda_{1,2,3}\right| \lesssim 4 \pi \text { and }\left|\frac{\lambda_{4}}{\Lambda}\right| \lesssim 4 \pi
$$

\subsection{Unitarity bounds}

The unitarity bound on the extended scalar sectors can be calculated from the scattering matrix (S-matrix) of different processes. The technique was developed in Refs. $[39,40]$ for the SM and it can also be applied to the HTM $(Y=0)$. The S-matrix for the HTM $(Y=0)$ consists of different scalarscalar, gauge boson-gauge boson, gauge boson-scalar scattering amplitudes. Using the Born approximation, the scattering cross-section for any process can be written as

$\sigma=\frac{16 \pi}{s} \sum_{l=1}^{\infty}(2 l+1)\left|a_{l}(s)\right|^{2}$,

where $s=4 E_{C M}^{2}$ is the Mandelstam variable, and $E_{C M}$ is the center of mass energy of the incoming particles. The $a_{l}$ are the partial wave coefficients corresponding to specific angular momenta $l$. This leads to the following unitarity constraint: $\operatorname{Re}\left(a_{l}\right)<\frac{1}{2}$. At high energy the dominant contribution to the amplitude $a_{l}$ of the two-body scattering processes $a, b \rightarrow c, d$ comes from the diagram involving the quartic couplings. Far away from the resonance, the other contributions to the amplitude from the scalar mediated $s-, t$-, and $u$-channel processes are negligibly small. Also, in the high energy limit, the amplitude of scattering processes involving longitudinal gauge bosons can be approximated by the scalar amplitude in which gauge bosons are replaced by their corresponding Goldstone bosons. For example, the amplitude of the $W_{L}^{+} W_{L}^{-} \rightarrow W_{L}^{+} W_{L}^{-}$scattering is equivalent to $G^{+} G^{-} \rightarrow G^{+} G^{-}$. This is known as the equivalence theorem [40-43]. So to test the unitarity of HTM $(Y=0)$, we construct the S-matrix which consists of only the scalar quartic couplings.
The scalar quartic couplings in the physical bases $G^{ \pm}, G^{0}$, $H^{ \pm}, h$ and $H$ are complicated functions of $\lambda^{\prime}$ s, $\gamma, \beta$. The hhhh vertex is $6\left(\lambda_{1} \cos ^{4} \gamma+\lambda_{3} \cos ^{2} \gamma \sin ^{2} \gamma+\lambda_{2} \sin ^{4} \gamma\right)$. It is difficult to calculate the unitary bounds in the physical bases. One can consider the non-physical scalar fields bases, i.e., $G_{1}^{ \pm}, \eta^{ \pm}, G^{0}, h^{0}$ and $\eta^{0}$ before the EWSB. Here the crucial point is that the S-matrix, which is expressed in terms of the physical fields, can be transformed into a S-matrix for the non-physical fields by making a unitary transformation $[44,45]$.

Different quartic couplings in non-physical bases are obtained by expanding the scalar potential of Eq. (2.6) which are given by

$$
\begin{aligned}
& \left\{G^{0} G^{0} G^{0} G^{0}\right\}=6 \lambda_{1}, \quad\left\{G_{1}^{+} G_{1}^{+} G_{1}^{-} G_{1}^{-}\right\}=4 \lambda_{1}, \\
& \left\{G_{1}^{+} G_{1}^{-} h^{0} h^{0}\right\}=2 \lambda_{1}, \quad\left\{G^{0} G^{0} \eta^{0} \eta^{0}\right\}=\lambda_{3}, \\
& \left\{h^{0} h^{0} \eta^{0} \eta^{0}\right\}=\lambda_{3}, \quad\left\{G^{0} G^{0} \eta^{+} \eta^{-}\right\}=\lambda_{3}, \\
& \left\{h^{0} h^{0} \eta^{+} \eta^{-}\right\}=\lambda_{3}, \quad\left\{G^{0} G^{0} G_{1}^{+} G_{1}^{-}\right\}=2 \lambda_{1}, \\
& \left\{G^{0} G^{0} h^{0} h^{0}\right\}=2 \lambda_{1}, \quad\left\{h^{0} h^{0} h^{0} h^{0}\right\}=6 \lambda_{1}, \\
& \left\{G_{1}^{+} G_{1}^{-} \eta^{0} \eta^{0}\right\}=\lambda_{3}, \quad\left\{\eta^{0} \eta^{0} \eta^{0} \eta^{0}\right\}=6 \lambda_{2}, \\
& \left\{G_{1}^{+} G_{1}^{-} \eta^{+} \eta^{-}\right\}=\lambda_{3}, \quad\left\{\eta^{0} \eta^{0} \eta^{+} \eta^{-}\right\}=2 \lambda_{2},
\end{aligned}
$$

$\left\{\eta^{+} \eta^{+} \eta^{-} \eta^{-}\right\}=4 \lambda_{2}$

The full set of these non-physical scalar scattering processes can be expressed as a $16 \times 16 \mathrm{~S}$-matrix. This matrix is composed of three submatrices of dimensions $6 \times 6,5 \times 5$, and $5 \times 5$ which have different initial and final states.

The first $6 \times 6$ sub-matrix, $\mathcal{M}_{1}$, corresponds to scattering processes whose initial and final states are one of $h^{0} G_{1}^{+}, G^{0} G_{1}^{+}, \eta^{0} G_{1}^{+}, h^{0} G_{1}^{+}, G^{0} \eta^{+}$, and $\eta^{0} \eta^{+}$. Using the Feynman rules in Eq. (3.5), one can obtain $\mathcal{M}_{1}$ $=\operatorname{diag}\left(2 \lambda_{1}, 2 \lambda_{1}, 2 \lambda_{1}, \lambda_{3}, \lambda_{3}, \lambda_{3}\right)$.

The sub-matrix $\mathcal{M}_{2}$ corresponds to scattering processes with one of the following initial and final states: $h^{0} G^{0}$, $G_{1}^{+} \eta^{-}, \eta^{+} G_{1}^{-}, \eta^{0} G^{0}$, and $h^{0} \eta^{0}$. Similarly, one can calculate $\mathcal{M}_{2}=\operatorname{diag}\left(2 \lambda_{1}, \lambda_{3}, \lambda_{3}, \lambda_{3}, \lambda_{3}\right)$.

The third sub-matrix, $\mathcal{M}_{3}$, corresponds to scattering fields $\left(G_{1}^{+} G_{1}^{-}, \eta^{+} \eta^{-}, \frac{G^{0} G^{0}}{\sqrt{2}}, \frac{h^{0} h^{0}}{\sqrt{2}}\right.$, and $\left.\frac{\eta^{0} \eta^{0}}{\sqrt{2}}\right)$. The factor $\frac{1}{\sqrt{2}}$ has appeared due to the statistics of identical particles. $\mathcal{M}_{3}$ is given by

$\mathcal{M}_{3}=\left(\begin{array}{ccccc}4 \lambda_{1} & \lambda_{3} & \sqrt{2} \lambda_{1} & \sqrt{2} \lambda_{1} & \frac{\lambda_{3}}{\sqrt{2}} \\ \lambda_{3} & 4 \lambda_{2} & \frac{\lambda_{3}}{\sqrt{2}} & \frac{\lambda_{3}}{\sqrt{2}} & \sqrt{2} \lambda_{2} \\ \sqrt{2} \lambda_{1} & \frac{\lambda_{3}}{\sqrt{2}} & 3 \lambda_{1} & \lambda_{1} & \frac{\lambda_{3}}{2} \\ \sqrt{2} \lambda_{1} & \frac{\lambda_{3}}{\sqrt{2}} & \lambda_{1} & 3 \lambda_{1} & \frac{\lambda_{3}}{2} \\ \frac{\lambda_{3}}{\sqrt{2}} & \sqrt{2} \lambda_{2} & \frac{\lambda_{3}}{2} & \frac{\lambda_{3}}{2} & 3 \lambda_{2}\end{array}\right)$. 
Eigenvalues of $\mathcal{M}_{3}$ are $2 \lambda_{1}, 2 \lambda_{1}, 2 \lambda_{2}$, and $\frac{1}{2}\left(6 \lambda_{1}+5 \lambda_{2} \pm\right.$ $\left.\sqrt{\left(6 \lambda_{1}-5 \lambda_{2}\right)^{2}+12 \lambda_{3}^{2}}\right)$.

Unitary constraints of the scattering processes demand that the eigenvalues of the S-matrix should be less than $8 \pi$.

\subsection{Bounds from electroweak precision experiments}

Electroweak precision data has imposed severe bounds on new physics models via the Peskin-Takeuchi parameters, $S, T, U$ [46]. The additional contributions from this model are given by $[21,26]$

$$
\begin{aligned}
S \simeq & 0, \\
T= & \frac{1}{8 \pi} \frac{1}{\sin ^{2} \theta_{W} \cos ^{2} \theta_{W}}\left[\frac{M_{H}^{2}+M_{H^{ \pm}}^{2}}{M_{Z}^{2}}\right. \\
& \left.-\frac{2 M_{H^{ \pm}}^{2} M_{H}^{2}}{M_{Z}^{2}\left(M_{H}^{2}-M_{H^{ \pm}}^{2}\right)} \log \left(\frac{M_{H}^{2}}{M_{H^{ \pm}}^{2}}\right)\right] \\
\simeq & \frac{1}{6 \pi} \frac{1}{\sin ^{2} \theta_{W} \cos ^{2} \theta_{W}} \frac{(\Delta M)^{2}}{M_{Z}^{2}} \\
U= & -\frac{1}{3 \pi}\left(M_{H}^{4} \log \left(\frac{M_{H}^{2}}{M_{H^{ \pm}}^{2}}\right) \frac{\left(3 M_{H^{ \pm}}^{2}-M_{H}^{2}\right)}{\left(M_{H}^{2}-M_{H^{ \pm}}^{2}\right)^{3}}\right. \\
& \left.+\frac{5\left(M_{H}^{4}+M_{H^{ \pm}}^{4}\right)-22 M_{H^{ \pm}}^{2} M_{H}^{2}}{6\left(M_{H}^{2}-M_{H^{ \pm}}^{2}\right)^{2}}\right) \\
\simeq & \frac{\Delta M}{3 \pi M_{H^{ \pm}}},
\end{aligned}
$$

where $\Delta M=M_{H^{ \pm}}-M_{H} . S$ is proportional to $\sin \beta$. The experimental value of the parameter $\rho$ demands the triplet VEV $v_{2}$ to be less than $4 \mathrm{GeV}$ [38]. Hence, the contributions to the $S$ parameter from the triplet scalar fields are negligible. $M_{H^{ \pm}}$and $M_{H}$ are almost degenerate for $M_{H^{ \pm}, H} \gg M_{h}$. The contributions to the parameters $T$ and $U$ from this model are also negligibly small [47].

\subsection{Bounds from LHC diphoton signal strength}

As the dominant production cross-section of $h$ at LHC is coming through gluon fusion, the Higgs to diphoton signal strength $\mu_{\gamma \gamma}$ can be written as

$$
\begin{aligned}
\mu_{\gamma \gamma} & =\frac{\sigma(g g \rightarrow h \rightarrow \gamma \gamma)_{H T M}}{\sigma(g g \rightarrow h \rightarrow \gamma \gamma)_{\mathrm{SM}}} \\
& =\frac{\sigma(g g \rightarrow h)_{H T M}}{\sigma(g g \rightarrow h)_{\mathrm{SM}}} \frac{B r(h \rightarrow \gamma \gamma)_{\mathrm{HTM}}}{B r(h \rightarrow \gamma \gamma)_{\mathrm{SM}}} .
\end{aligned}
$$

We use the narrow width approximation as $\Gamma_{h}^{\text {total }} / M_{h} \rightarrow 0$. The Higgs $h$ to $f \bar{f}$ and $V V$ ( $V$ stands for vector bosons) couplings are proportional to $\cos \gamma$, so they $\mu_{\gamma \gamma}$ can be simplified as $\mu_{\gamma \gamma}=\cos ^{2} \gamma \frac{\Gamma_{h, \mathrm{SM}}^{\mathrm{total}}}{\Gamma_{h, \mathrm{HTM}}^{\text {total }}} \frac{\Gamma(h \rightarrow \gamma \gamma)_{\mathrm{HTM}}}{\Gamma(h \rightarrow \gamma \gamma)_{\mathrm{SM}}}$.

The charged Higgs $H^{ \pm}$will alter the decay width of $h \rightarrow \gamma \gamma$, $Z \gamma$ through one loop, which implies $\Gamma(h \rightarrow \gamma \gamma, Z \gamma) \ll$ $\Gamma_{h}^{\text {total }}$. Also, if the mass of the extra scalar particles $(H T=$ $H, H^{ \pm}$) happen to be lighter than $M_{h} / 2$, then they might contribute to the invisible decay of the Higgs boson. Using the global fit analysis [48] we see that such an invisible branching ratio is less than $\sim 20 \%$. In Eq. (3.11), the first ratio provides a suppression of $\sim 0.8-1$. For $M_{H, H^{ \pm}}>M_{h} / 2$, the ratio becomes $\frac{\Gamma_{h, \mathrm{SM}}^{\mathrm{total}}}{\Gamma_{h, \mathrm{HTM}}^{\text {tota }}} \approx \frac{1}{\cos ^{2} \gamma}$. Hence, the Higgs to diphoton signal strength can be written as

$\mu_{\gamma \gamma} \approx \frac{\Gamma(h \rightarrow \gamma \gamma)_{\mathrm{HTM}}}{\Gamma(h \rightarrow \gamma \gamma)_{\mathrm{SM}}}$.

In HTM, the additional contributions to $\Gamma(h \rightarrow \gamma \gamma)$ at one loop due to the $H^{ \pm}$is given by [49]

$$
\begin{gathered}
\Gamma(h \rightarrow \gamma \gamma)_{\mathrm{HTM}}=\frac{\alpha^{2} M_{h}^{3}}{256 \pi^{3} v^{2}} \mid \sum_{f} N_{f}^{c} Q_{f}^{2} y_{f} F_{1 / 2}\left(\tau_{f}\right) \\
\quad+y_{W} F_{1}\left(\tau_{W}\right)+\left.Q_{H^{ \pm}}^{2} \frac{v \mu_{h H^{+} H^{-}}}{2 M_{H^{ \pm}}^{2}} F_{0}\left(\tau_{H^{ \pm}}\right)\right|^{2},
\end{gathered}
$$

where $\tau_{i}=M_{h}^{2} / 4 M_{i}^{2} . Q_{f}, Q_{H^{ \pm}}$denote the electric charges of the corresponding particles. $N_{f}^{c}$ is the color factor. $y_{f}$ and $y_{W}$ denote the Higgs couplings to $f \bar{f}$ and $W^{+} W^{-}$. $\mu_{h H^{+} H^{-}}=\left\{2 \lambda_{4} \sin \beta \cos \beta \cos \gamma+\cos \beta^{2}\left(\lambda_{3} v_{1} \cos \gamma+\right.\right.$ $\left.\left.4 \lambda_{2} v_{2} \sin \gamma\right)+\sin \beta^{2}\left(\lambda_{4} \sin \gamma+\lambda_{1} v_{1} \cos \gamma+\lambda_{3} v_{2} \sin \gamma\right)\right\} \approx$ $\lambda_{3} v_{\mathrm{SM}}$ stands for the coupling constant of the $h \mathrm{H}^{+} \mathrm{H}^{-}$vertex. The loop functions $F_{(0,1 / 2,1)}$ can be found in Ref [49].

Recently, the ATLAS [50] and CMS [51] collaborations have measured the ratio of the prediction of the diphoton rate $\mu_{\gamma \gamma}$ of the observed Higgs to the SM prediction. The present combined value of $\mu_{\gamma \gamma}$ is $1.14_{-0.18}^{+0.19}$ from these experiments [4].

In $\Gamma(h \rightarrow \gamma \gamma)_{\mathrm{HTM}}$ (see Eq. (3.13)), a positive $\lambda_{3}$ leads to a destructive interference between $H T$ and SM contributions and vice versa. One can see from Eq. (3.13) that the contribution to the Higgs diphoton channel is proportional to $\frac{\lambda_{3}}{M_{H^{ \pm}}^{2}}$. If the charged scalar mass is greater than $300 \mathrm{GeV}$, then the contribution of $H^{ \pm}$to the diphoton signal is negligibly small.

Now we present our results for the central values of the SM parameters such as the Higgs mass $M_{h}=125.7 \mathrm{GeV}$, the top mass $M_{t}=173.1 \mathrm{GeV}$, the $Z$ boson mass $M_{Z}=91.1876$ $\mathrm{GeV}$, and the strong coupling constant $\alpha_{\mathrm{s}}=0.1184$. We take the triplet vev $v_{2}, \lambda_{4}$ and the other quartic couplings $\lambda_{1,2,3}$ as input parameters. Hence, depending on these parameters the mixing angle $\gamma$ can vary in between 0 and $\pi / 2$. The triplet scalar masses also become arbitrarily heavy. Here, 


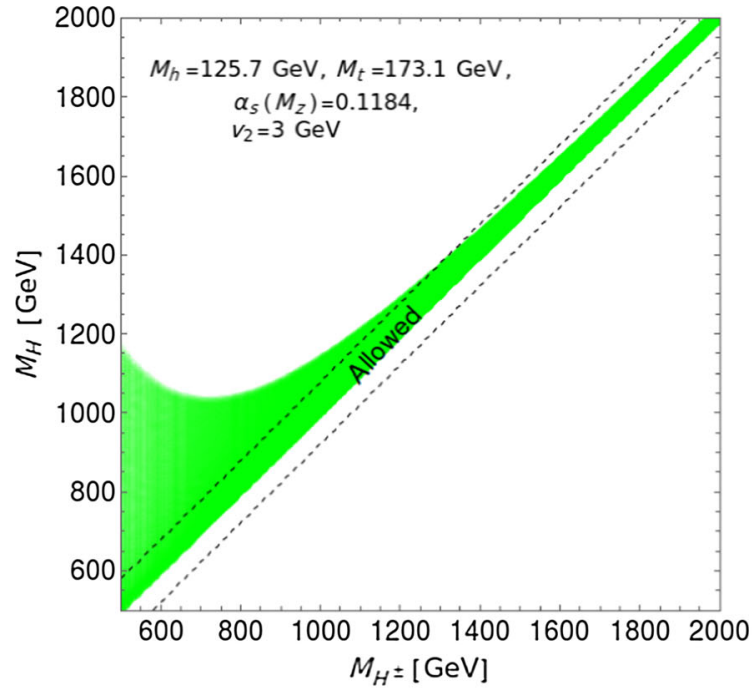

(a)

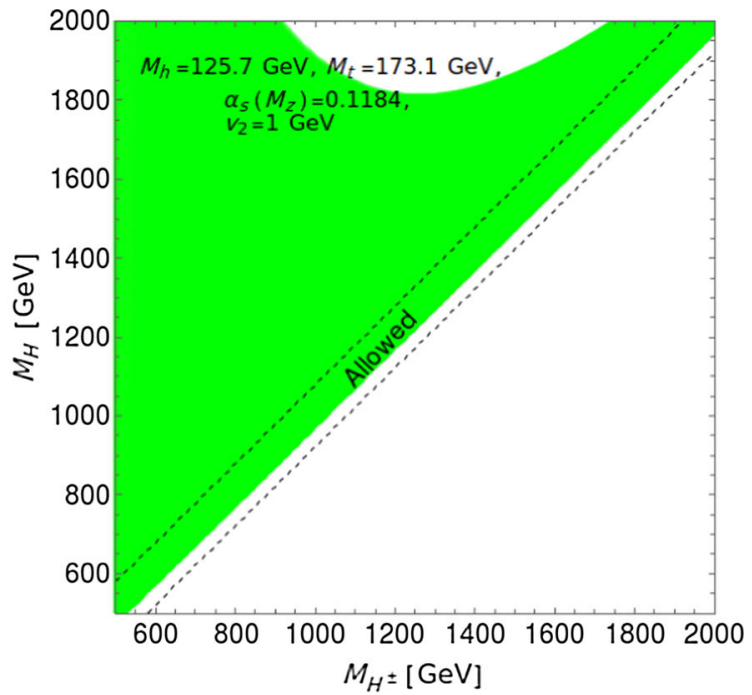

(b)

Fig. 1 The allowed region (green) from the unitarity, perturbativity and absolute stability which is valid up to the Planck mass $M_{\mathrm{Pl}}$. The region between the black-dashed line is allowed from the EWPT data at $2 \sigma$

we assume that no new physics shows up below the Planck mass $M_{\mathrm{Pl}}$. We examine the renormalization group (RG) flow of all couplings and establish bounds on the heavy scalar masses under the assumption that the parameters are valid up to the Planck mass $M_{\mathrm{Pl}}$. In this calculation, we use the SM RGEs up to three loops [52-55] and the triplet contributions up to two loops. We first calculate all couplings at $M_{t}$. To find their values at $M_{t}$, one needs to take into account different threshold corrections up to $M_{t}[5,6,15,16,76,77]$. Using the RGEs, we evolve all the coupling constants from $M_{t}$ to the Planck mass $M_{\mathrm{Pl}}$. By this procedure we obtain new parameter regions which are valid up to the Planck mass $M_{\mathrm{Pl}}$.

We show the allowed region (green) in the $M_{H^{ \pm}}-M_{H}$ plane for this model in Fig. 1. We demand that the EW vacuum of the scalar potential remain absolutely stable and do not violate the perturbative unitarity up to the Planck mass $M_{\mathrm{Pl}}$. One can also obtain the parameter spaces, corresponding to the metastable EW vacuum, which are seen to be small in this plane. Furthermore, we impose the EWPT constraints on the parameters so that the region between the black-dashed lines survives.

In Fig. 1, we show the allowed region for fixed central values of all the SM parameters. In the left panel, we present the plot for the choice of the quartic couplings $\lambda_{2,3}=0.1$ and triplet VEV $v_{2}=3 \mathrm{GeV}$. In the right panel, we use the value of the triplet VEV $v_{2}=1 \mathrm{GeV}$. We vary the quartic coupling $\lambda_{1}$ and dimensionful mass parameter $\lambda_{4}$ to calculate the neutral $C P$-even Higgs mass $M_{H}$, the charged Higgs mass $M_{H^{ \pm}}$and the mixing angle $\gamma$. These scalar masses increase, whereas the mixing angle decreases with $\lambda_{4}$. We find that the EW vacuum becomes unbounded from below for $\lambda_{1} \lesssim 0.128$. The theory also violates unitarity bounds for $\lambda_{1} \gtrsim 0.238$ before the Planck mass $M_{\mathrm{Pl}}$. One can see from Fig. 1a, the allowed region becomes smaller for the larger values of heavy scalar masses. In most of the parameter space the running couplings either violate unitary or perturbativity bounds before the Planck mass $M_{\mathrm{Pl}}$.

As $\lambda_{2,3}$ stabilize the scalar potential, we will get a wider green region for smaller scalar masses, but this will violate the unitarity bound in the higher mass region. We find that the EW vacuum becomes unbounded from below for the values of the quartic couplings $\lambda_{1} \lesssim 0.027$ and $\lambda_{2,3}=0.285$. We also check that the choice of the quartic couplings $\lambda_{1} \gtrsim$ 0.05 and $\lambda_{2,3}=0.285$ will violate unitary and perturbativity bounds before the Planck mass $M_{\mathrm{Pl}}$. One can also understand from the expressions of Eq. (2.13) that if we decrease the value of $v_{2}$, the area of the allowed region from the stability, unitary and perturbativity bounds will increase. We show the plot in Fig. $1 \mathrm{~b}$ for the choice of $v_{2}=1 \mathrm{GeV}$.

If the vacuum expectation value of the scalar triplet becomes zero, then the minimization condition of the scalar potential given in Eq. (2.8) is no longer valid. The mass parameter $\mu_{2}$ becomes free and the parameter $\lambda_{4}$ does not play any role in the stability analysis. In the next section, we will show the detailed stability analysis in the presence of extra $\mathbb{Z}_{2}$-symmetry in this model.

\section{Dark matter in HTM $(Y=0)$}

We impose a $Z_{2}$ symmetry on this model such that the scalar triplet is odd under this transformation, i.e., $T \rightarrow-T$, 


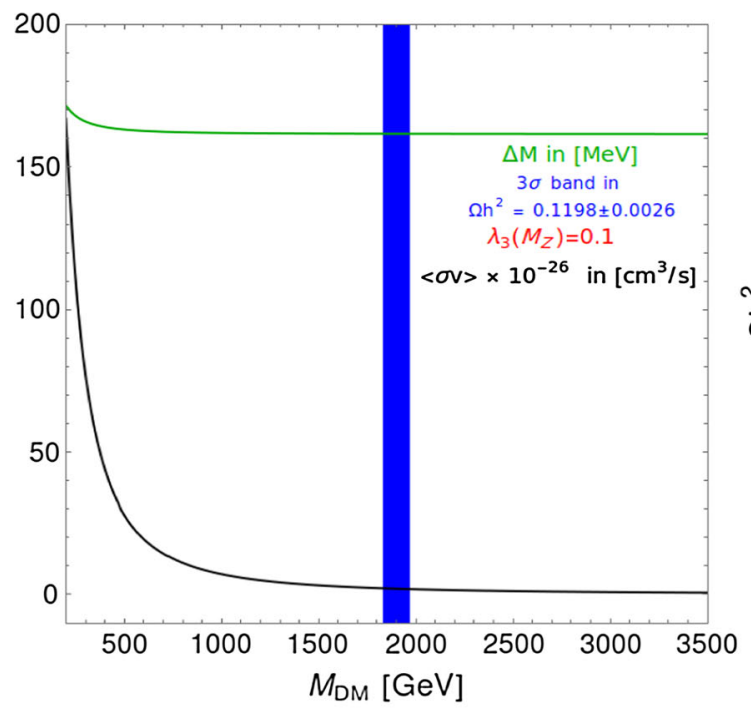

(a)

Fig. 2 The thin blue band corresponds to the relic density, $\Omega h^{2}=$ $0.1198 \pm 0.0026(3 \sigma)$ from the combined data of WMAP and Planck [63]. a The mass difference $\Delta M$ (green line) and the effective annihilation cross-section (black line) as a function of the dark matter

whereas the SM fields are even under this transformation. In the literature, the HTM including the $\mathbb{Z}_{2}$-symmetry is known as the inert triplet model (ITM) [31]. In this model, the term $\lambda_{4} H^{\dagger} \sigma^{a} \Phi T_{a}$ is absent in the scalar potential in Eq. (2.6), which implies $\lambda_{4}=0$. The $\mathbb{Z}_{2}$-symmetry prevents the triplet scalar to acquire a VEV, i.e., $v_{2}=0$. The potential can have a minimum along the Higgs field direction only. The EWSB is driven by the SM Higgs doublet. The scalar fields of the triplet do not mix with the scalar fields of SM doublet. After the EWSB, the scalar potential in Eq. (2.6) is then given by

$$
\begin{aligned}
& V\left(h, H, H^{ \pm}\right) \\
& =\frac{1}{4}\left[2 \mu_{1}^{2}(h+v)^{2}+\lambda_{1}(h+v)^{4}+2 \mu_{2}^{2}\left(H^{2}+2 H^{+} H^{-}\right)\right. \\
& \left.\quad+\lambda_{2}\left(H^{2}+2 H^{+} H^{-}\right)^{2}+\lambda_{3}(h+v)^{2}\left(H^{2}+2 H^{+} H^{-}\right)\right] .
\end{aligned}
$$

Here, $v \equiv v_{\mathrm{SM}}$ and the masses (see Eq. (2.9)) of the scalar fields ${ }^{1} h, H$ and $H^{ \pm}$are given by

$M_{h}^{2}=2 \lambda_{1} v^{2}$,

$M_{H}^{2}=\mu_{2}^{2}+\frac{\lambda_{3}}{2} v^{2}$,

$M_{H^{ \pm}}^{2}=\mu_{2}^{2}+\frac{\lambda_{3}}{2} v^{2}$.

At tree level the mass of the neutral scalar $H$ and the charged particles $H^{ \pm}$are degenerate. If we include a one-loop

1 For $v_{2}=0$, the notation in Eq. (2.1) $H \equiv \eta^{0}$ and $H^{ \pm} \equiv \eta^{ \pm}$are the physical scalar fields.

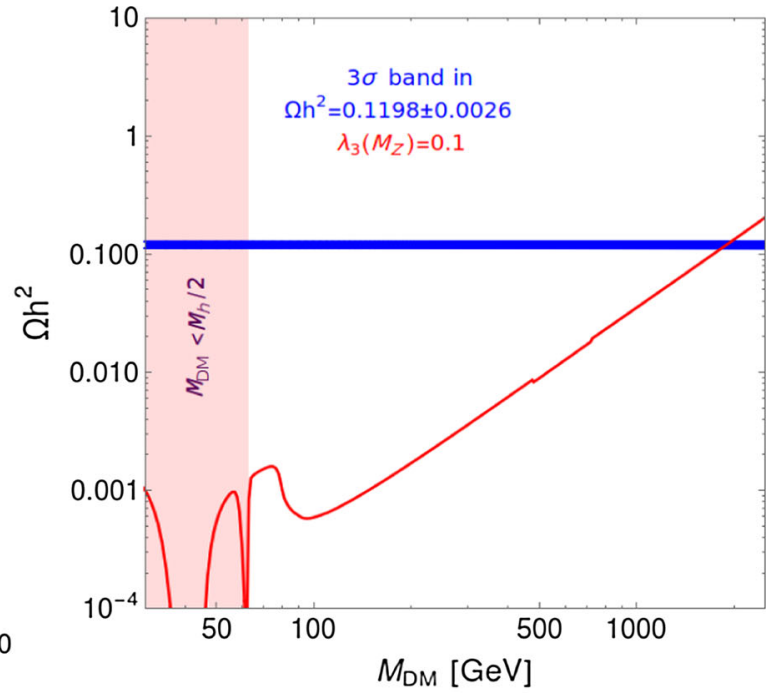

(b)

mass for the portal coupling $\lambda_{3}\left(M_{Z}\right)=0.10$. b The relic density $\Omega h^{2}$ as a function of the DM mass $M_{\mathrm{DM}}\left(\equiv M_{H}\right)$ (red line) for $\lambda_{3}\left(M_{Z}\right)=0.10$

radiative correction, the charged particles become slightly heavier [56,57] than the neutral ones. The mass difference between them is given by

$$
\begin{aligned}
\Delta M & =\left(M_{H^{ \pm}}-M_{H}\right)_{1 \text {-loop }} \\
& =\frac{\alpha M_{H}}{4 \pi}\left[f\left(\frac{M_{W}}{M_{H}}\right)-c_{W}^{2} f\left(\frac{M_{Z}}{M_{H}}\right)\right],
\end{aligned}
$$

with $f(x)=-\frac{x}{4}\left\{2 x^{3} \log (x)+\left(x^{2}-4\right)^{\frac{3}{2}} \log \left(\frac{x^{2}-2-x \sqrt{x^{2}-4}}{2}\right)\right\}$. It has been shown in Refs. [56,57] that the mass splitting between charged and neutral scalars remains $\sim 150$ $\mathrm{MeV}$ for $M_{H}=0.1-5 \mathrm{TeV}$. In Fig. 2a, we show the variation $\Delta M$ (green line) with the $M_{H}$ (三 $\left.M_{\mathrm{DM}}\right)$ mass. As the $\mathbb{Z}_{2}$-symmetry also prohibits the couplings of an odd number of scalar fields of the triplet with the SM particles, $H$ can serve as a viable DM candidate which may saturate the measured DM relic density of the Universe. In this work, we use the software package FeynRules [60] along with micrOMEGAs $[61,62]$ to calculate the relic density of the DM. As $\Delta M$ is very small, the effective annihilation cross-section is dominated by the co-annihilation channels $H H^{ \pm} \rightarrow$ SM particles [59]. Although it is dominated by the co-annihilation channel, we need a very small Higgs portal coupling $\lambda_{3}$ to obtain the correct relic density. The effective annihilation cross-section (see the black line in Fig. 2a) decreases rapidly with $\Delta M$ for the DM mass below $500 \mathrm{GeV}$ and becomes $\sim 10^{-26} \mathrm{~cm}^{3} s^{-1}$ around $M_{\mathrm{DM}}=2000 \mathrm{GeV}$. We obtain the relic density in the right ballpark. 
In Fig. 2b, we present the plot for the relic density as a function of the DM mass for the fixed Higgs portal coupling $\lambda_{3}\left(M_{Z}\right)=0.10$. The light-red band is excluded from the Higgs invisible decay width [58]. There are two deep regions in the relic density band (red line). The first one is situated near the DM mass $M_{\mathrm{DM}} \approx 45 \mathrm{GeV}$. It is due the resonance of the $s$-channel $H H^{ \pm} \rightarrow \mathrm{SM}$ fermions processes, mediated by the vector bosons $W^{ \pm}$. The second one is situated near the DM mass $M_{\mathrm{DM}} \approx M_{h} / 2$ for the Higgs-mediated $H H \rightarrow$ $\mathrm{SM}$ fermions processes. There is another, shallower region located around the DM mass $M_{\mathrm{DM}}=100 \mathrm{GeV}$, which is due to the dominant contributions coming from $\mathrm{HH}^{ \pm}, \mathrm{HH} \rightarrow$ gauge bosons channels.

For $500 \mathrm{GeV}$, we find that the total cross-section $\langle\sigma v\rangle \sim$ $10^{-25} \mathrm{~cm}^{3} \mathrm{~s}^{-1}$, so the relic density becomes $\sim 0.01$. In this region, the dominant channels are $H, H^{ \pm} \rightarrow Z W^{ \pm}, \gamma W^{ \pm}$ $(\sim 35, \sim 10 \%)$ and $H^{ \pm}, H^{ \pm} \rightarrow Z W^{ \pm}(\sim 25 \%)$. We also check that the smaller dark matter mass along with the Higgs portal coupling $\lambda_{3}$ (within the perturbative limit) does alter the relic density only in the third decimal place. If we increase the DM masses, then the effective annihilation cross-section decreases. This is mainly due to the mass suppression. We get a DM relic density in the right ballpark for DM masses greater than $1.8 \mathrm{TeV}$.

One can see that the mass splitting $\Delta M$ attains saturation for $M_{\mathrm{DM}}>700 \mathrm{GeV}$. Hence, the relic density is mainly regulated by the Higgs-mediated $s$-channel processes, although the contributions are small. We check that the Higgs portal coupling $\lambda_{3}$ can be varied in between 0 to 1 for the DM mass $1850-2200 \mathrm{GeV}$ to get the right relic density. For example, we obtain the relic density $\Omega h^{2}=0.1198$ for $\lambda_{3}=0.001$ and $M_{\mathrm{DM}}=1894.5 \mathrm{GeV}$. We get the same relic density for $\lambda_{3}=0.8$ and $M_{\mathrm{DM}}=2040 \mathrm{GeV}$. However, the running couplings will violate the unitary and perturbativity bounds for $\lambda_{3} \gtrsim 0.6$.

The non-observation of DM signals in direct detection experiments at XENON 100 [64,65], LUX [66] and LUX2016 [67] put severe restrictions [33] on the Higgs portal coupling $\lambda_{3}$ for a given DM mass. In this model, we check the parameter regions which are satisfying the relic density and are allowed by the recent LUX-2016 [67] and XENON1T2017 [68] data.

\subsection{Metastability in ITM $(Y=0)$}

As in the SM the EW vacuum is metastable, it is important to explore if ITM has any solution in its reserve. As the scalar WIMP $H$ protected by $\mathbb{Z}_{2}$-symmetry can serve as a viable DM candidate, it is interesting to explore if they help prolong the lifetime of the Universe. The effective Higgs potential gets modified in the presence of these new extra scalars.

The one-loop effective Higgs potential in $\overline{\mathrm{MS}}$ scheme and the Landau gauge is given by
$V_{1}^{\mathrm{SM}+\mathrm{IT}}(h)=V_{1}^{\mathrm{SM}}(h)+V_{1}^{\mathrm{IT}}(h)$,

where [69-73]

$V_{1}^{\mathrm{SM}}(h)=\sum_{i=1}^{5} \frac{n_{i}}{64 \pi^{2}} M_{i}^{4}(h)\left[\ln \frac{M_{i}^{2}(h)}{\mu^{2}(t)}-c_{i}\right]$.

$n_{i}$ is the number of degrees of freedom and $M_{i}^{2}(h)=$ $\kappa_{i}(t) h^{2}(t)-\kappa_{i}^{\prime}(t) . n_{i}, c_{i}, \kappa_{i}$ and $\kappa_{i}^{\prime}$ can be found in Eq. (4) in Ref. [69]. $t$ is a dimensionless parameter which is expressed in terms of the running parameter $\mu(t)=M_{Z} \exp (t)$.

The contributions to the effective Higgs potential from the new scalars $\left(H, H^{ \pm}\right)$of the inert scalar triplet are given by [21]

$V_{1}^{\mathrm{IT}}(h)=\sum_{j=H, H^{+}, H^{-}} \frac{1}{64 \pi^{2}} M_{j}^{4}(h)\left[\ln \left(\frac{M_{j}^{2}(h)}{\mu^{2}(t)}\right)-\frac{3}{2}\right]$,

where $M_{j}^{2}(h)=\frac{1}{2} \lambda_{j}(t) h^{2}(t)+\mu_{2}^{2}(t)$, with $\lambda_{H, H^{ \pm}}(t)=$ $\lambda_{3}(t)$. In the present work, in the Higgs effective potential, SM contributions are taken up to two-loop level $[5,6,74,75]$ and the IT scalar contributions are considered up to one loop only [21].

For $h \gg v$, the quantum corrections to the Higgs potential are reabsorbed in the effective running coupling $\lambda_{1 \text {,eff }}$, so that the effective potential becomes

$V_{\mathrm{eff}}^{\mathrm{SM}+\mathrm{IT}}(h) \simeq \lambda_{1, \mathrm{eff}}(h) \frac{h^{4}}{4}$,

with

$\lambda_{1, \mathrm{eff}}(h)=\lambda_{1, \mathrm{eff}}^{\mathrm{SM}}(h)+\lambda_{1, \mathrm{eff}}^{\mathrm{IT}}(h)$,

where the expression of $\lambda_{1, \text { eff }}^{\mathrm{SM}}(h)$ up to two-loop quantum corrections can be found in Ref. [5] and $\lambda_{1, \text { eff }}^{\mathrm{IT}}(h)=$ $e^{4 \Gamma(h)}\left[\frac{3 \lambda_{3}^{2}}{256 \pi^{2}}\left(\ln \left(\frac{\lambda_{3}}{2}\right)-\frac{3}{2}\right)\right]$, with $\Gamma(h)=\int_{M_{t}}^{h} \gamma(\mu) d$ $\ln \mu$. The wave function renormalization of the Higgs field is taken into account by the anomalous dimension $\gamma(\mu)$. Here, all running coupling constants are evaluated at $\mu=h$, ensuring that the potential remains within the perturbative domain.

We first calculate all couplings with the threshold corrections $[5,6,15,16,76,77]$ at $M_{t}$. Then we evolve all the couplings up to the Planck mass $M_{\mathrm{Pl}}$ using our own computer codes incorporating the RG equations. Here, the SM effects in the RGEs are taken up to three loops [52-55] and IT contributions are considered up to two loops (see Appendix A).

We choose a specific benchmark point $M_{\mathrm{DM}}\left(\equiv M_{H}\right)=$ $1897 \mathrm{GeV}, M_{h}=125.7 \mathrm{GeV}$ and $\alpha_{\mathrm{s}}\left(M_{Z}\right)=0.1184$, so that it can give the right DM density of the Universe. The corresponding values of all quartic couplings $\lambda_{1,2,3}$ at $M_{t}=$ $173.1 \mathrm{GeV}$ and $M_{\mathrm{Pl}}=1.2 \times 10^{19} \mathrm{GeV}$ are presented in Table 1. For this benchmark point, we show the evolution of the running of the quartic couplings $\left(\lambda_{1,2,3}\right)$ in Fig. 3 . We 
Table 1 A set of values of all quartic coupling constants at $M_{t}$ and $M_{\mathrm{Pl}}$ for $M_{\mathrm{DM}}=1897 \mathrm{GeV}$

\begin{tabular}{llll}
\hline & $\lambda_{1}$ & $\lambda_{2}$ & $\lambda_{3}$ \\
\hline$M_{t}$ & 0.127054 & 0.10 & 0.10 \\
$M_{\mathrm{Pl}}$ & -0.00339962 & 0.267706 & 0.206306 \\
\hline
\end{tabular}

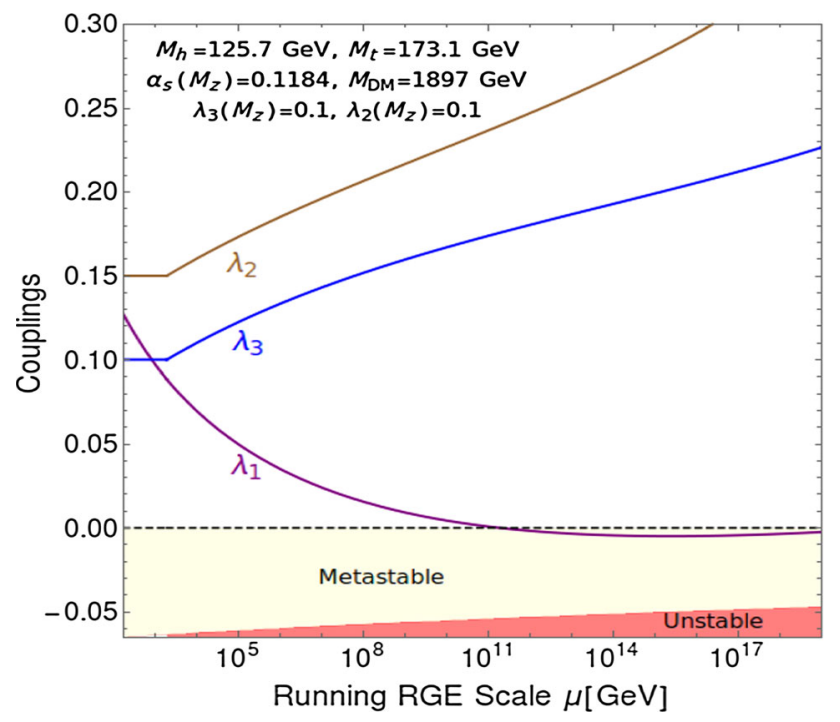

Fig. 3 RG evolution of the couplings $\lambda_{1,2,3}$ for the set of parameters in Table 1 with DM mass $M_{\mathrm{DM}}=1897 \mathrm{GeV}$

find that this specific choice of benchmark point with the top mass ${ }^{2} M_{t}=173.1 \mathrm{GeV}$ and the central values of other SM parameters leads to a metastable EW vacuum. It implies that the $\beta$ function of the Higgs quartic coupling $\lambda_{1}$ becomes zero at very high energy scale and remains positive up to the Planck mass $M_{\mathrm{Pl}}$. We find that a deeper minimum is situated at that high energy scale before the Planck mass $M_{\mathrm{Pl}}$. We also check that the EW vacuum remains metastable (one-sided) for the quartic coupling $\lambda_{2} \leq 0.1$, the Higgs portal coupling $\lambda_{3} \leq 0.15$ and the DM mass $M_{\mathrm{DM}} \geq 1900 \mathrm{GeV}$. We obtain the stable EW vacuum ( $>99.99 \%$ confidence level, onesided) for the choice of the parameters $\lambda_{2}=0.1, \lambda_{3}=0.3$ and $M_{\mathrm{DM}}=1915 \mathrm{GeV}$. The running couplings will violate the unitary and perturbativity bounds for $\lambda_{3} \gtrsim 0.6$. In the following subsections, we will discuss the metastability of the EW vacuum of the scalar potential.

\subsection{Tunneling probability}

Using the experimentally measured values of the SM parameters at the EW scale, when analyzing the SM scalar potential at higher energy scales, one encounters the so-called metasta-

2 As the $\beta$ function of the Higgs quartic coupling, $\lambda_{1}$ contains $-\frac{6 y_{t}^{4}}{16 \pi^{2}}$ (see Eq. (A1)), the values of the Higgs quartic couplings $\lambda_{1}$ at very high energies are extremely sensitive to $M_{t}$. bility of the EW vacuum $[5-7,15,16]$. Since a second (true) minimum, deeper than the EW minimum, is situated near the Planck mass, there exists a non-zero probability that the EW minimum will tunnel into the second minimum. The tunneling probability of the EW vacuum to the true vacuum at the present epoch can be expressed as $[5,78,79]$

$\mathcal{P}_{0}=0.15 \frac{\Lambda_{B}^{4}}{H^{4}} e^{-S\left(\Lambda_{B}\right)}$,

where $S\left(\Lambda_{B}\right)$ is the minimum action of the Higgs potential of a bounce of size $R=\Lambda_{B}^{-1}$ and is given by

$S\left(\Lambda_{B}\right)=\frac{8 \pi^{2}}{3\left|\lambda_{1}\left(\Lambda_{B}\right)\right|}$.

It becomes minimum when $\lambda_{1}\left(\Lambda_{B}\right)$ is minimum, i.e., $\beta_{\lambda_{1}}\left(\Lambda_{B}\right)=0$. In this work, we neglect loop [78] and gravitational corrections $[80,81]$ to the action as in Refs. $[15,16]$. A finite temperature also affects to EW vacuum stability $[78,82,83]$. In this work, we consider field theory in the zero-temperature limit.

In the ITM, the additional scalar fields give a positive contribution to $\beta_{\lambda_{1}}$ (see Eqs. (A1) and (A2). Due to the presence of these extra scalars, a metastable EW vacuum goes towards the stability, i.e., the tunneling probability $\mathcal{P}_{0}$ becomes smaller. We first calculate the minimum value of $\lambda_{1, \text { eff }}$ of Eq. (4.8). Putting this minimum value in Eq. (4.10), we compute the tunneling probability $\mathcal{P}_{0}$. As the stability of the EW vacuum is very sensitive to the top mass $M_{t}$, we show the variation of the tunneling probability $\mathcal{P}_{0}$ as a function of $M_{t}$ in Fig. 4a. The right band in Fig. 4a corresponds to the tunneling probability for our benchmark point. We present $\mathcal{P}_{0}$ for the SM as the left band to see the effect of the additional IT scalar. We also display $1 \sigma$ error bands in $\alpha_{\mathrm{S}}$ (light-gray) and $M_{h}$ (light-red). One can see from this figure that the effect of $\alpha_{\mathrm{s}}$ on the tunneling probability is larger than the effect of $M_{h}$. To see the effect of the ITM parameter spaces, we plot $\mathcal{P}_{0}$ as a function of the Higgs portal coupling $\lambda_{3}\left(M_{Z}\right)$ in Fig. $4 \mathrm{~b}$ for different choices of $\lambda_{2}\left(M_{Z}\right)$. We keep the fixed central values of all SM parameters. Here, the DM mass $M_{\mathrm{DM}}$ is also varied with $\lambda_{3}$ to get the DM relic density $\Omega h^{2}=0.1198$.

The additional IT scalar fields in the IT model improve the stability of the EW vacuum as follows:

- If $0>\lambda_{1}\left(\Lambda_{B}\right)>\lambda_{1, \min }\left(\Lambda_{B}\right)$, then the vacuum is metastable.

- If $\lambda_{1}\left(\Lambda_{B}\right)<\lambda_{1, \min }\left(\Lambda_{B}\right)$, then the vacuum is unstable.

- If $\lambda_{2}<0$, the potential is unbounded from below along the $H$ and $H^{ \pm}$-direction.

- If $\lambda_{3}\left(\Lambda_{\mathrm{I}}\right)<0$, the potential is unbounded from below along a direction in between $H$ and $h$ and also $H^{ \pm}$and $h$. 


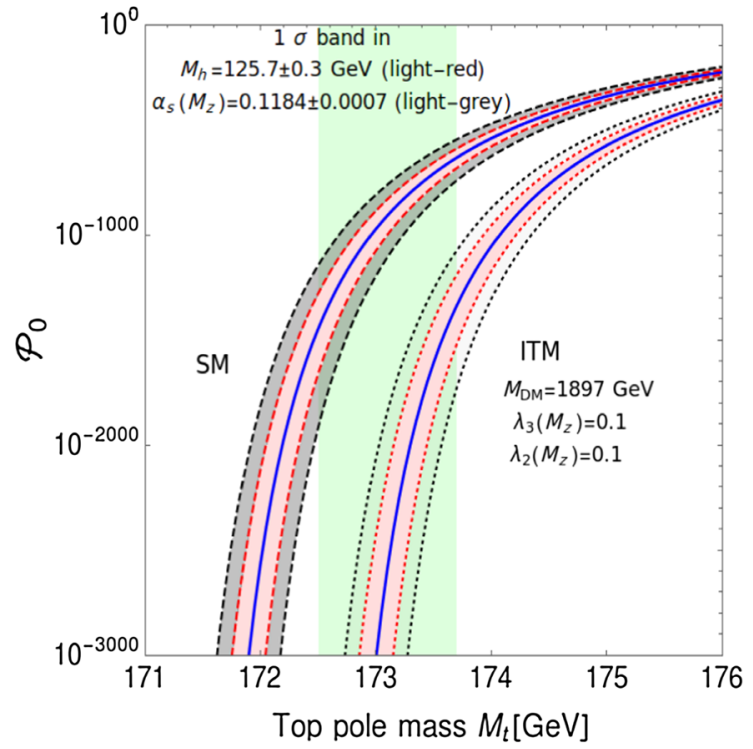

(a)

Fig. 4 a Tunneling probability $\mathcal{P}_{0}$ dependence on $M_{t}$. The left band (between dashed lines) corresponds to the SM. The right one (between dotted lines) is for IT model for a DM mass $M_{H}=1897 \mathrm{GeV}$. Dark

In the above $\lambda_{1, \min }\left(\Lambda_{B}\right)=\frac{-0.06488}{1-0.00986 \ln \left(v / \Lambda_{B}\right)}$ and $\Lambda_{\mathrm{I}}$ represents any energy scale for which $\lambda_{1}$ is negative $[15,16]$.

\subsection{Phase diagrams}

In order to show the explicit dependence of the electroweak stability for different parameters of the ITM, we present various kinds of phase diagrams.

In Fig. 5a, we calculate the confidence level for our bench mark points $M_{\mathrm{DM}}=1897 \mathrm{GeV}, \lambda_{2}\left(M_{Z}\right)=0.10$ and $\lambda_{3}\left(M_{Z}\right)=0.10$ by drawing an ellipse passing through the stability line $\lambda=\beta_{\lambda}=0$ in the $M_{t}-M_{h}$ plane. If the area of the ellipse is $\chi$ times the area of the ellipse, it represents the $1 \sigma$ error in the same plane. This factor $\chi$ is the confidence level of the stability of EW vacuum. We develop a proper method to calculate this factor and the tangency point for the stability line. In this case, the confidence level of metastability is decreased (one-sided) with $\alpha_{\mathrm{S}}\left(M_{Z}\right)$, i.e., the EW vacuum moves towards the stability region. We obtain the similar factor in the $\alpha_{\mathrm{S}}\left(M_{Z}\right)-M_{t}$ plane. In this case, the confidence level decreases with $M_{h}$. One can see from the phase diagrams in Fig. 5 that the stable EW vacuum is excluded at $1.2 \sigma$ (one-sided).

If the ITM is valid up to the Planck mass, which also saturates the DM abundance of the Universe, then the confidence level vs. $\lambda_{3}\left(M_{Z}\right)$ phase diagram becomes important to realize where the present EW vacuum is residing. In Fig. 6, we vary the DM mass with $\lambda_{3}\left(M_{Z}\right)$ to keep the relic density at $\Omega h^{2}=0.1198$. One can see that the EW vacuum approaches the stability with larger values of $\lambda_{2,3}\left(M_{Z}\right)$. The

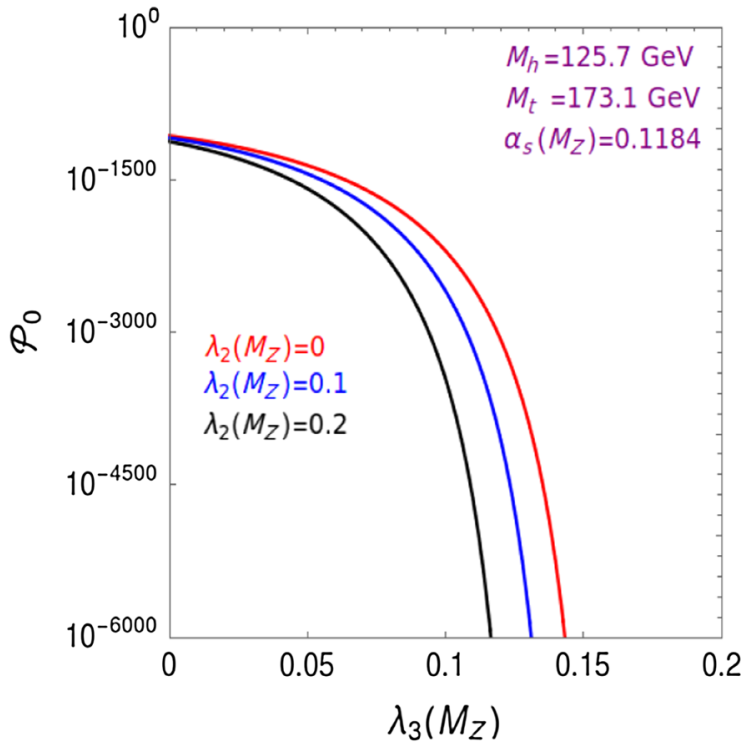

(b)

matter constraints are respected for these specific choice of parameters. The light-green band stands for $M_{t}$ at $\pm 1 \sigma$. b $\mathcal{P}_{0}$ is plotted against the Higgs DM coupling $\lambda_{3}\left(M_{Z}\right)$ for different values of $\lambda_{2}\left(M_{Z}\right)$

EW vacuum becomes absolutely stable for $\lambda_{3}\left(M_{Z}\right) \geq 0.154$ and $\lambda_{2}\left(M_{Z}\right) \approx 0.10$ (see the blue line in Fig. 6). We show this phase diagram for central values of the SM parameters. Moreover, if we increase the top mass and/or decrease the Higgs mass along with $\alpha_{\mathrm{s}}\left(M_{Z}\right)$, then the size of the region corresponding to the metastable EW vacuum will increase. We see that the conditions of a DM mass $M_{\mathrm{DM}} \geq 1912 \mathrm{GeV}$, $\lambda_{3}\left(M_{Z}\right) \geq 0.31$ and $\lambda_{2}\left(M_{Z}\right) \geq 0.1$ are required to stabilize the EW vacuum for $M_{t}=174.9 \mathrm{GeV}, M_{h}=124.8 \mathrm{GeV}$ and $\alpha_{\mathrm{s}}\left(M_{Z}\right)=0.1163$.

In Fig. 7, we show the allowed parameter spaces in the $\lambda_{3}\left(M_{Z}\right)-M_{H^{ \pm}}$plane for central values of the SM parameters and $\lambda_{2}\left(M_{Z}\right)=0.1$. The lower (red) region is excluded since the scalar potential becomes unbounded from below along the direction in between $H^{ \pm}$and $h$. In this region, the effective Higgs quartic coupling is negative and at the same time $\lambda_{3}$ remains negative up to the Planck mass $M_{\mathrm{Pl}}$. We obtain the parameter space with negative $\lambda_{3}\left(M_{Z}\right)$, which is also allowed from metastability. In this case, $\lambda_{3}$ becomes positive at the scale $\Lambda_{B}$ and remains positive up to the Planck mass $M_{\mathrm{Pl}}$. The EW vacuum is absolutely stable in the green region. The upper red region violates unitary bounds. The right side of the black dotted line is allowed from $\mu_{\gamma \gamma}$ at $1 \sigma$.

\section{Inflation in HTM $(Y=0)$}

Observations of super-horizon ansiotropies in the CMB data, measured by various experiments such as WMAP and Planck, have established that the early Universe underwent 


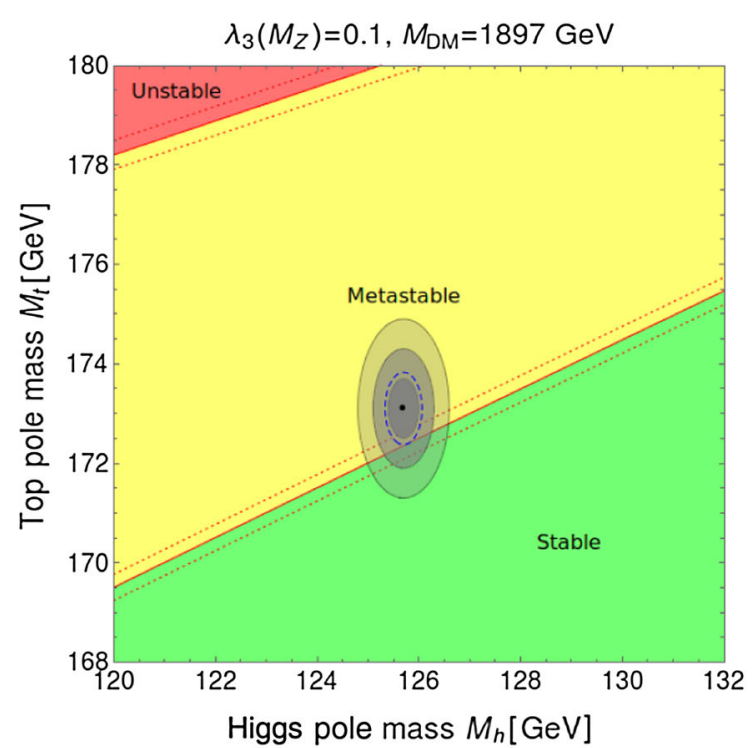

(a)

Fig. 5 Phase diagrams in a the $M_{h}-M_{t}$ plane and $\mathbf{b}$ the $M_{t}-\alpha_{\mathrm{S}}\left(M_{Z}\right)$ plane ITM. Regions of absolute stability (green), metastability (yellow), instability (red) of the EW vacuum are also marked. The gray zones rep-

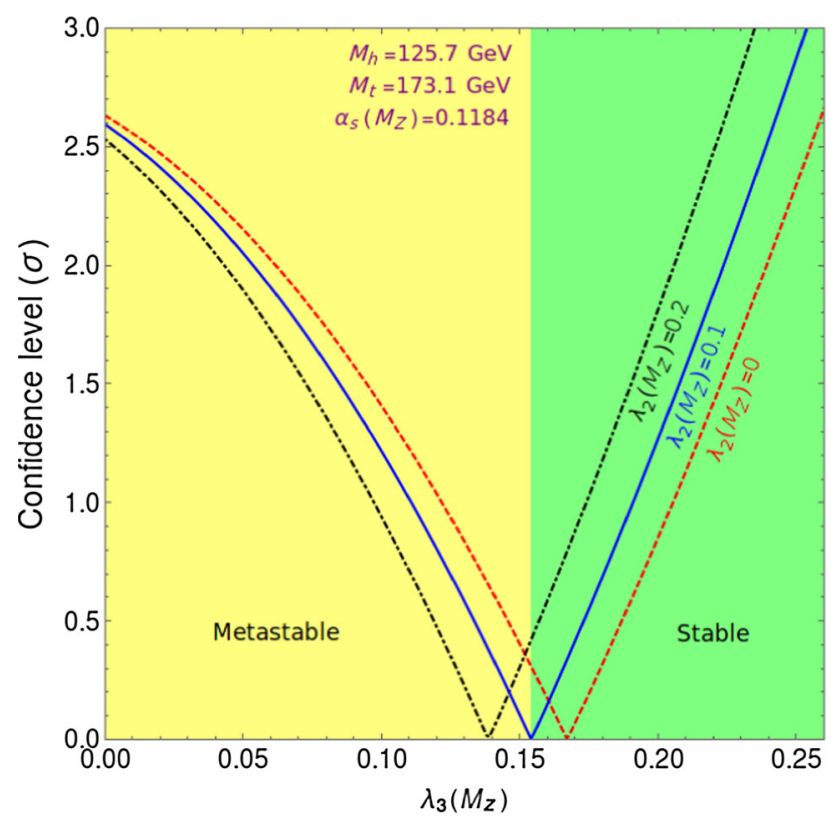

Fig. 6 Dependence of confidence level at which the EW vacuum stability is excluded (one-sided) or allowed on $\lambda_{3}\left(M_{Z}\right)$ and $\lambda_{2}\left(M_{Z}\right)$ in ITM. Regions of absolute stability (green) and metastability (yellow) of EW vacuum are shown for $\lambda_{2}\left(M_{Z}\right)=0.1$

a period of rapid expansion. This is known as inflation. This can solve a number of cosmological problems, such as the horizon problem, the flatness problem and the magnetic monopole problem of the present Universe. If the electroweak vacuum is metastable, then the Higgs is unlikely to play the role of an inflaton [84-92] in the SM. Therefore,

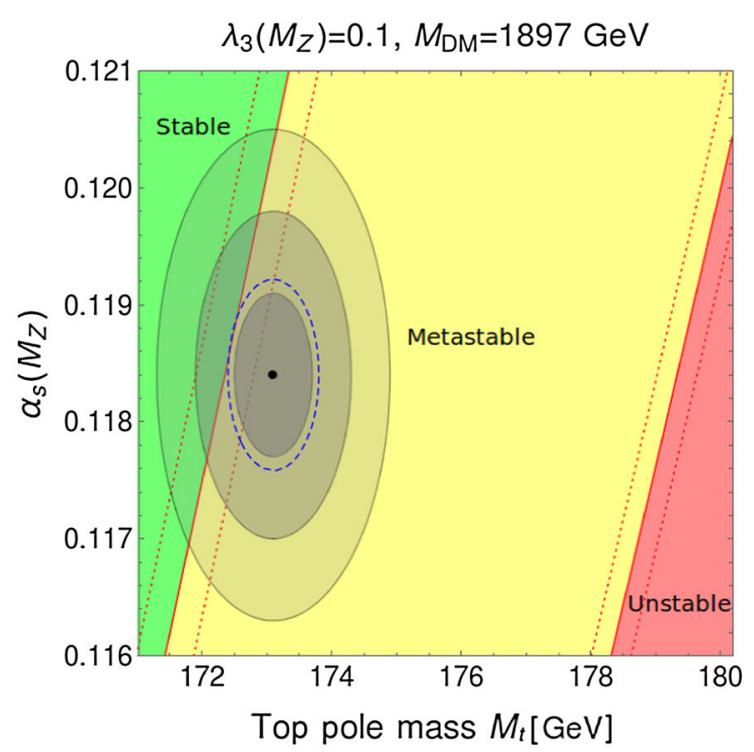

(b)

resent error ellipses at 1,2 and $3 \sigma$. The three boundary lines (dotted, solid and dotted red) correspond to $\alpha_{\mathrm{s}}\left(M_{Z}\right)=0.1184 \pm 0.0007$

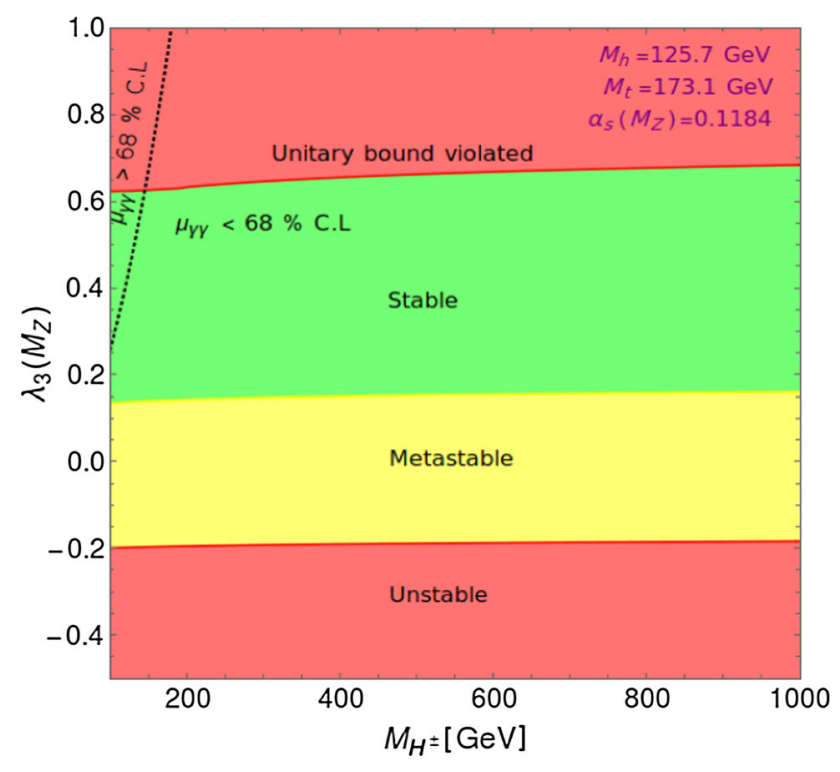

Fig. 7 Phase diagram in $\lambda_{3}\left(M_{Z}\right)-M_{H^{ \pm}}$plane in ITM. The right side of the black dotted line is allowed from the signal strength ratio of $\mu_{\gamma \gamma}$ within $68 \%$ confidence level and the left side is excluded at $1 \sigma$. In the metastable region, the Higgs portal coupling $\lambda_{3}\left(M_{Z}\right)$ is negative; however, beyond the scale $\Lambda_{B}$ it is greater than zero

extra new degrees of freedom are needed in addition to the SM ones to explain inflation in the early Universe [93-98].

Here, we study an extension of the Higgs sector with a real triplet scalar $T$ in the presence of large couplings $\zeta_{h, H}$ to the Ricci scalar curvature $R$. This theory can explain inflation in the early Universe at the large field values in the scale invariance Einstein frame. 
In this model, the action of the fields in a Jordan frame is given by

$$
\begin{aligned}
S_{j}= & \int \sqrt{-g} d^{4} x\left[\mathcal{L}_{\mathrm{SM}}+\frac{1}{2}\left(\partial_{\mu} \Phi\right)^{\dagger}\left(\partial_{\mu} \Phi\right)+\frac{1}{2}\left(\partial_{\mu} T\right)^{\dagger}\left(\partial_{\mu} T\right)\right. \\
& \left.-\zeta_{h} R|\Phi|^{2}-\zeta_{H} R|T|^{2}-V(\Phi, T)\right] .
\end{aligned}
$$

In the present work, we consider $H$ as an inflaton. The Higgs $h$ can also act as an inflaton for the stable EW vacuum. In order to calculate the inflationary observables such as the tensor-to-scalar ratio $r$, the spectral index $n_{\mathrm{S}}$ and the running of the spectral index $n_{\mathrm{rs}}$, we perform a conformal transformation from Jordan frame to Einstein frame, so that the nonminimal coupling $\zeta_{H}$ of the scalar field to the Ricci scalar disappears.

The transformation is given by [99]

$$
\tilde{g}_{\mu \nu}=\Omega^{2} g_{\mu \nu}, \quad \Omega=\sqrt{1+\zeta_{H} \frac{H^{2}}{M_{\mathrm{Pl}}^{2}}} .
$$

The action of Eq. (5.1) in the Einstein frame can be written as

$S=\int \sqrt{-g} d^{4} x\left[\frac{1}{2}\left(\partial_{\mu} \chi\right)^{\dagger}\left(\partial_{\mu} \chi\right)-V(\chi)\right]$,

where

$\frac{\mathrm{d} \chi}{\mathrm{d} H}=\sqrt{\frac{\Omega^{2} M_{\mathrm{Pl}}^{2}+6 \zeta_{H}^{2} H^{2}}{\Omega^{4} M_{\mathrm{Pl}}^{2}}}$.

The scalar potential $V(\chi)$ is then given by

$V(\chi)=\lambda_{2} \frac{M_{\mathrm{Pl}}^{4}}{4 \zeta_{H}^{2}}\left(1+\exp \left(-\sqrt{\frac{2 \chi}{3 M_{\mathrm{Pl}}}}\right)\right)^{-2}$.

We plot this potential in Fig. 8 for the choice of the bench mark point $\zeta_{H}=1$ and $\lambda_{2}=10^{-9}$. One can also get the same plot for the parameters $\zeta_{H}=10^{4}$ and $\lambda_{2}=0.1$. However, this choice of the parameters violates the unitary bound. One can see that the potential has the ability to explain slow-roll inflation.

One can define the slow-roll parameters $\epsilon, \eta$ and $\zeta$ in terms of the potential by

$\epsilon=\frac{1}{2}\left(\frac{1}{V} \frac{\mathrm{d} V}{\mathrm{~d} \chi}\right)^{2}, \quad \eta=\frac{1}{V} \frac{\mathrm{d}^{2} V}{\mathrm{~d} \chi^{2}}, \quad$ and $\zeta=\frac{1}{V^{2}} \frac{\mathrm{d} V}{\mathrm{~d} \chi} \frac{\mathrm{d}^{3} V}{\mathrm{~d} \chi^{3}}$

The inflationary observable quantities such as the tensor-toscalar ratio $r$, the spectral index $n_{\mathrm{S}}$ and the running of the spectral index $n_{\text {rs }}$ are defined as

$r=16 \epsilon, \quad n_{\mathrm{s}}=1-6 \epsilon+2 \eta, \quad$ and

$n_{\mathrm{rs}}=-2 \zeta-24 \epsilon^{2}+16 \eta \epsilon$

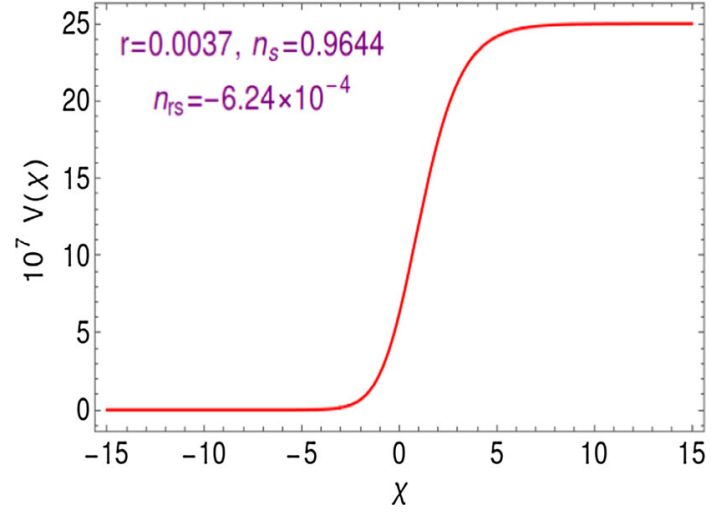

Fig. 8 Inflation potential in the Planck unit for $\zeta_{H}=1$ and $\lambda_{2}=10^{-9}$

and the number of $e$-folds is given by

$N=\int_{\chi_{\text {start }}}^{\chi_{\text {end }}} \frac{V}{\mathrm{~d} V / \mathrm{d} \chi} \mathrm{d} \chi$

where $\chi_{\text {start }}\left(\chi_{\text {end }}\right)$ is the initial (final) value when inflation starts (ends). At $\chi_{\text {start }}, \epsilon$ is 1 . We calculate the $\chi_{\text {end }}$ from Eq. (5.7) for $N=60$.

At the end of inflation, we get

$r=0.0037, \quad n_{\mathrm{s}}=0.9644, \quad$ and $n_{\mathrm{rs}}=-6.24 \times 10^{-4}$,

which is allowed by the present experimental data at $1 \sigma[100$, 101]. Hence, the neutral component of the triplet scalar can simultaneously serve as an inflaton and dark matter particle as well.

\section{Discussion and conclusions}

The measurements of the properties of the Higgs-like scalar boson detected at the Large Hadron Collider on 4th July 2012 are consistent with the minimal choice of the scalar sector. But the experimental data of the Higgs signal strengths and the uncertainties in the measurement of other standard model parameters still allow for an extended scalar sector. We have taken an extra hyperchargeless scalar triplet as new physics. First, we have assumed that the extra neutral $C P$-even component of the scalar triplet has also participated in the EWSB. We have shown the detailed structure of the tree-level scalar potential and mixing of the scalar fields. We have also discussed the bounds on the VEV $\left(v_{2}\right)$ of the neutral $C P$-even component of the scalar triplet from the $\rho$-parameter. To the best of our knowledge the full expressions of the unitary bounds on the quartic couplings of the scalar potential in this model have not yet been presented in the literature. We have shown these unitary bounds in this model. As the SM gauge symmetry $S U(2)_{L}$ prohibits the coupling of SM neu- 
trinos with the neutral $C P$-even component $\left(\eta^{0}\right)$ of the scalar triplet, the model does not lead to neutrino masses. But the model is still interesting, as it can play a role in improving the stability of the Higgs potential. We have taken into account various threshold corrections to calculate all the couplings at $M_{t}$. Then using three-loop SM RGEs and two-loop triplet RGEs, we have evolved all the couplings up to the Planck mass $M_{\mathrm{Pl}}$. We have shown the allowed region in the $M_{H^{ \pm-}}$ $M_{H}$ plane. We have demanded that the EW vacuum of the scalar potential remain absolutely stable and do not violate the perturbative unitarity up to the Planck mass $M_{\mathrm{Pl}}$. We have discussed the constraints on the parameter spaces from the recent LHC $\mu_{\gamma \gamma}$ and $\mu_{Z \gamma}$ data. Furthermore, only a very small region of the parameter space is shown to survive on imposing the EWPT constraints.

Astrophysical observations of various kinds, such as anomalies in the galactic rotation curves and gravitational lensing effects in bullet clusters, have indicated the existence of DM in the Universe. In the ITM, the extra scalar fields are protected by a discrete $\mathbb{Z}_{2}$-symmetry which ensures the stability of the lightest neutral particle. We have verified that the mass of the neutral scalar particle $(H)$ is slightly lighter than the mass of the charged particle $\left(H^{ \pm}\right)$so that the contributions coming from co-annihilation between $\mathrm{H}$ and $H^{ \pm}$ play a significant role in the relic density calculation. In the low mass region, the co-annihilation rates are quite high so that the dark matter density is found to be much smaller than the right relic density $\Omega h^{2}=0.1198 \pm 0.0026$ of the Universe. We have obtained the relic density in the right ballpark for a DM mass greater than $1.8 \mathrm{TeV}$. In this context, we have shown how the presence of an additional hyperchargeless scalar triplet improves the stability of the Higgs potential. In this study, we have used state of the art next-to-next-to leading order (NNLO) for the SM calculations. We have used the SM Higgs scalar potential up to two-loop quantum corrections which is improved by three-loop renormalization groups of the SM couplings. We have taken into account the contributions to the effective Higgs potential of the new scalars at one loop only. These contributions are improved by two-loop renormalization groups of the new parameters. In this paper, we have explored the stability of the EW minimum of the new effective Higgs potential up to the Planck mass $M_{\mathrm{Pl}}$. We have presented new modified stability conditions for the metastable EW vacuum. We have also shown various phase diagrams in various parameter spaces to show the explicit dependence of the EW (meta)stability on various parameters. For the first time, we have identified new regions of parameter space that correspond to the stable and metastable EW vacuum, which also provides the relic density of the DM in the Universe as measured by the WMAP and Planck experiments. In the present paper, we have also shown that the extra neutral scalar field $H$ can play a role in inflation and can serve as a dark matter candidate. The scalar potential can explain inflation for large scalar field values. We have obtained the inflationary observables as observed by the experiments.

Acknowledgements This work is supported by a fellowship from the University Grants Commission. This work is partially supported by a Grant from the Department of Science and Technology, India, via Grant no. EMR/2014/001177. I would like to thank Subhendu Rakshit, Amitava Raychaudhuri, Amitava Datta, Subhendra Mohanty and Girish K. Chakravarty for useful discussions.

Open Access This article is distributed under the terms of the Creative Commons Attribution 4.0 International License (http://creativecomm ons.org/licenses/by/4.0/), which permits unrestricted use, distribution, and reproduction in any medium, provided you give appropriate credit to the original author(s) and the source, provide a link to the Creative Commons license, and indicate if changes were made. Funded by SCOAP ${ }^{3}$.

\section{Appendix A: Two-loop beta functions for IT model}

In this study, we use the SM RGEs up to three loops which have been given in Refs. [52-55]. The triplet contributions $\left(\lambda_{2,3}\right)$ are taken up to two loops which have been generated using SARAH $[102,103]$.

In the HTM $(Y=0)$, the RGEs of the couplings $\left(\chi_{i}=\right.$ $g_{1,2,3}, \lambda_{1,2,3}$ and $\left.Y_{l, u, d}\right)$ and dimensionful mass parameters $\left(\mu_{1,2}\right.$ and $\left.\lambda_{4}\right)$ are defined as

$\beta_{\chi_{i}}=\frac{\partial \chi_{i}}{\partial \ln \mu}=\frac{1}{16 \pi^{2}} \beta_{\chi_{i}}^{(1)}+\frac{1}{\left(16 \pi^{2}\right)^{2}} \beta_{\chi_{i}}^{(2)}$.

For $\mu>M_{H}$, the RGEs of the scalar quartic couplings $\lambda_{1,2,3}$ and the mass parameter $\lambda_{4}$ are given by

$$
\begin{aligned}
\beta_{\lambda_{1}}^{(1)}= & +\frac{27}{200} g_{1}^{4}+\frac{9}{20} g_{1}^{2} g_{2}^{2}+\frac{9}{8} g_{2}^{4}-\frac{9}{5} g_{1}^{2} \lambda_{1}-9 g_{2}^{2} \lambda_{1}+24 \lambda_{1}^{2} \\
& +\frac{3}{2} \lambda_{3}^{2}+12 \lambda_{1} \operatorname{Tr}\left(Y_{d} Y_{d}^{\dagger}\right)+4 \lambda_{1} \operatorname{Tr}\left(Y_{l} Y_{l}^{\dagger}\right) \\
& +12 \lambda_{1} \operatorname{Tr}\left(Y_{u} Y_{u}^{\dagger}\right)-6 \operatorname{Tr}\left(Y_{d} Y_{d}^{\dagger} Y_{d} Y_{d}^{\dagger}\right) \\
& -2 \operatorname{Tr}\left(Y_{l} Y_{l}^{\dagger} Y_{l} Y_{l}^{\dagger}\right)-6 \operatorname{Tr}\left(Y_{u} Y_{u}^{\dagger} Y_{u} Y_{u}^{\dagger}\right), \\
\beta_{\lambda_{1}}^{(2)}= & -\frac{3411}{2000} g_{1}^{6}-\frac{1677}{400} g_{1}^{4} g_{2}^{2}-\frac{317}{80} g_{1}^{2} g_{2}^{4}+\frac{277}{16} g_{2}^{6}+\frac{1887}{200} g_{1}^{4} \lambda_{1} \\
& +\frac{117}{20} g_{1}^{2} g_{2}^{2} \lambda_{1}-\frac{29}{8} g_{2}^{4} \lambda_{1}+\frac{108}{5} g_{1}^{2} \lambda_{1}^{2} \\
& +108 g_{2}^{2} \lambda_{1}^{2}-312 \lambda_{1}^{3}+5 g_{2}^{4} \lambda_{3}+12 g_{2}^{2} \lambda_{3}^{2}-15 \lambda_{1} \lambda_{3}^{2}-2 \lambda_{3}^{3} \\
& +\frac{1}{20}\left(-5\left(64 \lambda_{1}\left(-5 g_{3}^{2}+9 \lambda_{1}\right)-90 g_{2}^{2} \lambda_{1}+9 g_{2}^{4}\right)\right. \\
& \left.+9 g_{1}^{4}+g_{1}^{2}\left(50 \lambda_{1}+54 g_{2}^{2}\right)\right) \operatorname{Tr}\left(Y_{d} Y_{d}^{\dagger}\right) \\
& -\frac{3}{20}\left(15 g_{1}^{4}-2 g_{1}^{2}\left(11 g_{2}^{2}+25 \lambda_{1}\right)\right. \\
& \left.+5\left(-10 g_{2}^{2} \lambda_{1}+64 \lambda_{1}^{2}+g_{2}^{4}\right)\right) \\
& \operatorname{Tr}\left(Y_{l} Y_{l}^{\dagger}\right)-\frac{171}{100} g_{1}^{4} \operatorname{Tr}\left(Y_{u} Y_{u}^{\dagger}\right)
\end{aligned}
$$




$$
\begin{aligned}
& +\frac{63}{10} g_{1}^{2} g_{2}^{2} \operatorname{Tr}\left(Y_{u} Y_{u}^{\dagger}\right)-\frac{9}{4} g_{2}^{4} \operatorname{Tr}\left(Y_{u} Y_{u}^{\dagger}\right) \\
& +\frac{17}{2} g_{1}^{2} \lambda_{1} \operatorname{Tr}\left(Y_{u} Y_{u}^{\dagger}\right)+\frac{45}{2} g_{2}^{2} \lambda_{1} \operatorname{Tr}\left(Y_{u} Y_{u}^{\dagger}\right) \\
& +80 g_{3}^{2} \lambda_{1} \operatorname{Tr}\left(Y_{u} Y_{u}^{\dagger}\right)-144 \lambda_{1}^{2} \operatorname{Tr}\left(Y_{u} Y_{u}^{\dagger}\right) \\
& +\frac{4}{5} g_{1}^{2} \operatorname{Tr}\left(Y_{d} Y_{d}^{\dagger} Y_{d} Y_{d}^{\dagger}\right)-32 g_{3}^{2} \operatorname{Tr}\left(Y_{d} Y_{d}^{\dagger} Y_{d} Y_{d}^{\dagger}\right) \\
& -3 \lambda_{1} \operatorname{Tr}\left(Y_{d} Y_{d}^{\dagger} Y_{d} Y_{d}^{\dagger}\right)-42 \lambda_{1} \operatorname{Tr}\left(Y_{d} Y_{u}^{\dagger} Y_{u} Y_{d}^{\dagger}\right) \\
& -\frac{12}{5} g_{1}^{2} \operatorname{Tr}\left(Y_{l} Y_{l}^{\dagger} Y_{l} Y_{l}^{\dagger}\right)-\lambda_{1} \operatorname{Tr}\left(Y_{l} Y_{l}^{\dagger} Y_{l} Y_{l}^{\dagger}\right) \\
& -\frac{8}{5} g_{1}^{2} \operatorname{Tr}\left(Y_{u} Y_{u}^{\dagger} Y_{u} Y_{u}^{\dagger}\right)-32 g_{3}^{2} \operatorname{Tr}\left(Y_{u} Y_{u}^{\dagger} Y_{u} Y_{u}^{\dagger}\right) \\
& -3 \lambda_{1} \operatorname{Tr}\left(Y_{u} Y_{u}^{\dagger} Y_{u} Y_{u}^{\dagger}\right)+30 \operatorname{Tr}\left(Y_{d} Y_{d}^{\dagger} Y_{d} Y_{d}^{\dagger} Y_{d} Y_{d}^{\dagger}\right) \\
& +6 \operatorname{Tr}\left(Y_{d} Y_{d}^{\dagger} Y_{d} Y_{u}^{\dagger} Y_{u} Y_{d}^{\dagger}\right)-12 \operatorname{Tr}\left(Y_{d} Y_{u}^{\dagger} Y_{u} Y_{d}^{\dagger} Y_{d} Y_{d}^{\dagger}\right) \\
& -6 \operatorname{Tr}\left(Y_{d} Y_{u}^{\dagger} Y_{u} Y_{u}^{\dagger} Y_{u} Y_{d}^{\dagger}\right) \\
& +10 \operatorname{Tr}\left(Y_{l} Y_{l}^{\dagger} Y_{l} Y_{l}^{\dagger} Y_{l} Y_{l}^{\dagger}\right)+30 \operatorname{Tr}\left(Y_{u} Y_{u}^{\dagger} Y_{u} Y_{u}^{\dagger} Y_{u} Y_{u}^{\dagger}\right), \\
& \beta_{\lambda_{2}}^{(1)}=2\left(11 \lambda_{2}^{2}-12 g_{2}^{2} \lambda_{2}+3 g_{2}^{4}+\lambda_{3}^{2}\right) \\
& \beta_{\lambda_{2}}^{(2)}=-\frac{272}{3} g_{2}^{6}+\frac{94}{3} g_{2}^{4} \lambda_{2}+160 g_{2}^{2} \lambda_{2}^{2}-244 \lambda_{2}^{3}+10 g_{2}^{4} \lambda_{3} \\
& +\frac{12}{5} g_{1}^{2} \lambda_{3}^{2}+12 g_{2}^{2} \lambda_{3}^{2}-20 \lambda_{2} \lambda_{3}^{2}-8 \lambda_{3}^{3} \\
& -12 \lambda_{3}^{2} \operatorname{Tr}\left(Y_{d} Y_{d}^{\dagger}\right)-4 \lambda_{3}^{2} \operatorname{Tr}\left(Y_{l} Y_{l}^{\dagger}\right)-12 \lambda_{3}^{2} \operatorname{Tr}\left(Y_{u} Y_{u}^{\dagger}\right), \\
& \beta_{\lambda_{3}}^{(1)}=+3 g_{2}^{4}-\frac{9}{10} g_{1}^{2} \lambda_{3}-\frac{33}{2} g_{2}^{2} \lambda_{3}+12 \lambda_{1} \lambda_{3}+10 \lambda_{2} \lambda_{3} \\
& +4 \lambda_{3}^{2}+6 \lambda_{3} \operatorname{Tr}\left(Y_{d} Y_{d}^{\dagger}\right)+2 \lambda_{3} \operatorname{Tr}\left(Y_{l} Y_{l}^{\dagger}\right) \\
& +6 \lambda_{3} \operatorname{Tr}\left(Y_{u} Y_{u}^{\dagger}\right) \\
& \beta_{\lambda_{3}}^{(2)}=-\frac{9}{4} g_{1}^{2} g_{2}^{4}+\frac{329}{12} g_{2}^{6}+30 g_{2}^{4} \lambda_{1}+20 g_{2}^{4} \lambda_{2} \\
& +\frac{1671}{400} g_{1}^{4} \lambda_{3}+\frac{9}{8} g_{1}^{2} g_{2}^{2} \lambda_{3}-\frac{607}{48} g_{2}^{4} \lambda_{3}+\frac{72}{5} g_{1}^{2} \lambda_{1} \lambda_{3} \\
& +72 g_{2}^{2} \lambda_{1} \lambda_{3}-60 \lambda_{1}^{2} \lambda_{3}+88 g_{2}^{2} \lambda_{2} \lambda_{3}-34 \lambda_{2}^{2} \lambda_{3} \\
& +\frac{3}{5} g_{1}^{2} \lambda_{3}^{2}+11 g_{2}^{2} \lambda_{3}^{2}-72 \lambda_{1} \lambda_{3}^{2}-52 \lambda_{2} \lambda_{3}^{2} \\
& -\frac{23}{2} \lambda_{3}^{3}+\left(-12 \lambda_{3}^{2}-3 g_{2}^{4}+40 g_{3}^{2} \lambda_{3}-72 \lambda_{1} \lambda_{3}\right. \\
& \left.+\frac{45}{4} g_{2}^{2} \lambda_{3}+\frac{5}{4} g_{1}^{2} \lambda_{3}\right) \operatorname{Tr}\left(Y_{d} Y_{d}^{\dagger}\right) \\
& -\frac{1}{4}\left(4 g_{2}^{4}+\lambda_{3}\left(16 \lambda_{3}+96 \lambda_{1}-15 g_{2}^{2}-15 g_{1}^{2}\right)\right) \operatorname{Tr}\left(Y_{l} Y_{l}^{\dagger}\right) \\
& -3 g_{2}^{4} \operatorname{Tr}\left(Y_{u} Y_{u}^{\dagger}\right)+\frac{17}{4} g_{1}^{2} \lambda_{3} \operatorname{Tr}\left(Y_{u} Y_{u}^{\dagger}\right) \\
& +\frac{45}{4} g_{2}^{2} \lambda_{3} \operatorname{Tr}\left(Y_{u} Y_{u}^{\dagger}\right)+40 g_{3}^{2} \lambda_{3} \operatorname{Tr}\left(Y_{u} Y_{u}^{\dagger}\right) \\
& -72 \lambda_{1} \lambda_{3} \operatorname{Tr}\left(Y_{u} Y_{u}^{\dagger}\right)-12 \lambda_{3}^{2} \operatorname{Tr}\left(Y_{u} Y_{u}^{\dagger}\right) \\
& -\frac{27}{2} \lambda_{3} \operatorname{Tr}\left(Y_{d} Y_{d}^{\dagger} Y_{d} Y_{d}^{\dagger}\right)-21 \lambda_{3} \operatorname{Tr}\left(Y_{d} Y_{u}^{\dagger} Y_{u} Y_{d}^{\dagger}\right)
\end{aligned}
$$

$$
\begin{aligned}
& -\frac{9}{2} \lambda_{3} \operatorname{Tr}\left(Y_{l} Y_{l}^{\dagger} Y_{l} Y_{l}^{\dagger}\right)-\frac{27}{2} \lambda_{3} \operatorname{Tr}\left(Y_{u} Y_{u}^{\dagger} Y_{u} Y_{u}^{\dagger}\right) \\
\beta_{\lambda_{4}}^{(1)}= & 2 \lambda_{4} \operatorname{Tr}\left(Y_{l} Y_{l}^{\dagger}\right)+4 \lambda_{1} \lambda_{4}+4 \lambda_{3} \lambda_{4}+6 \lambda_{4} \operatorname{Tr}\left(Y_{d} Y_{d}^{\dagger}\right) \\
& +6 \lambda_{4} \operatorname{Tr}\left(Y_{u} Y_{u}^{\dagger}\right)-\frac{21}{2} g_{2}^{2} \lambda_{4}-\frac{9}{10} g_{1}^{2} \lambda_{4}, \\
\beta_{\lambda_{4}}^{(2)}= & +\frac{1311}{400} g_{1}^{4} \lambda_{4}+\frac{141}{40} g_{1}^{2} g_{2}^{2} \lambda_{4}-\frac{1343}{48} g_{2}^{4} \lambda_{4} \\
& +\frac{24}{5} g_{1}^{2} \lambda_{1} \lambda_{4}-28 \lambda_{1}^{2} \lambda_{4}+5 \lambda_{2}^{2} \lambda_{4}+\frac{3}{5} g_{1}^{2} \lambda_{3} \lambda_{4} \\
& +23 g_{2}^{2} \lambda_{3} \lambda_{4}-40 \lambda_{1} \lambda_{3} \lambda_{4}-20 \lambda_{2} \lambda_{3} \lambda_{4} \\
& -\frac{17}{2} \lambda_{3}^{2} \lambda_{4}+\frac{\lambda_{4}}{4}\left(160 g_{3}^{2}+45 g_{2}^{2}-48 \lambda_{3}+5 g_{1}^{2}\right. \\
& \left.-96 \lambda_{1}\right) \operatorname{Tr}\left(Y_{d} Y_{d}^{\dagger}\right)+\frac{1}{4}\left(15 g_{1}^{2}+15 g_{2}^{2}\right. \\
& \left.-16\left(2 \lambda_{1}+\lambda_{3}\right)\right) \lambda_{4} \operatorname{Tr}\left(Y_{l} Y_{l}^{\dagger}\right) \\
& +\frac{17}{4} g_{1}^{2} \lambda_{4} \operatorname{Tr}\left(Y_{u} Y_{u}^{\dagger}\right)+\frac{45}{4} g_{2}^{2} \lambda_{4} \operatorname{Tr}\left(Y_{u} Y_{u}^{\dagger}\right) \\
& +40 g_{3}^{2} \lambda_{4} \operatorname{Tr}\left(Y_{u} Y_{u}^{\dagger}\right)-24 \lambda_{1} \lambda_{4} \operatorname{Tr}\left(Y_{u} Y_{u}^{\dagger}\right) \\
& -12 \lambda_{3} \lambda_{4} \operatorname{Tr}\left(Y_{u} Y_{u}^{\dagger}\right)-\frac{27}{2} \lambda_{4} \operatorname{Tr}\left(Y_{d} Y_{d}^{\dagger} Y_{d} Y_{d}^{\dagger}\right) \\
& +27 \lambda_{4} \operatorname{Tr}\left(Y_{d} Y_{u}^{\dagger} Y_{u} Y_{d}^{\dagger}\right)-\frac{9}{2} \lambda_{4} \operatorname{Tr}\left(Y_{l} Y_{l}^{\dagger} Y_{l} Y_{l}^{\dagger}\right) \\
& \frac{27}{2} \lambda_{4} \operatorname{Tr}\left(Y_{u} Y_{u}^{\dagger} Y_{u} Y_{u}^{\dagger}\right) \\
& \\
& \\
&
\end{aligned}
$$

For $\mu<M_{H}, \beta_{\lambda_{1}}=\beta_{\lambda_{1}}\left(\lambda_{2,3}=0\right)$ and $\beta_{\lambda_{2,3,4}}=0$, where $Y_{u}=y_{u}, y_{c}, y_{t}$ are the Yukawa couplings of up, charm and top quark, $Y_{d}=y_{d}, y_{\mathrm{s}}, y_{b}$ for down, strange and bottom quark. $Y_{l}$ represents the Yukawa couplings for the charged leptons. In our work, we have included the contribution only from the top quark. Since the other Yukawa couplings are very small, they do not alter our result. We have also taken into account the contributions to the beta functions of the gauge couplings $g_{1,2,3}$ of the new physics. The importance of the mass parameters $\mu_{1,2}$ and $\lambda_{4}$ are found to be negligible in the stability analysis.

\section{References}

1. G. Aad et al., ATLAS Collaboration. Phys. Lett. B 716, 1 (2012). arXiv:1207.7214 [hep-ex]

2. S. Chatrchyan et al., CMS Collaboration. Phys. Lett. B 716, 30 (2012). arXiv:1207.7235 [hep-ex]

3. P.P. Giardino, K. Kannike, I. Masina, M. Raidal, A. Strumia, JHEP 1405, 046 (2014). arXiv:1303.3570 [hep-ph]

4. G. Aad et al., ATLAS and CMS Collaborations. JHEP 1608, 045 (2016). arXiv:1606.02266 [hep-ex]

5. D. Buttazzo, G. Degrassi, P.P. Giardino, G.F. Giudice, F. Sala, A. Salvio, A. Strumia, JHEP 1312, 089 (2013). arXiv:1307.3536

6. G. Degrassi, S. Di Vita, J. Elias-Miro, J.R. Espinosa, G.F. Giudice, G. Isidori, A. Strumia, JHEP 1208, 098 (2012). arXiv:1205.6497 [hep-ph] 
7. I. Masina, Phys. Rev. D 87, 053001 (2013). arXiv: 1209.0393 [hep$\mathrm{ph}]$

8. J. Elias-Miro, J.R. Espinosa, G.F. Giudice, G. Isidori, A. Riotto, A. Strumia, Phys. Lett. B 709, 222 (2012). arXiv:1112.3022 [hep$\mathrm{ph}]$

9. V. Branchina, E. Messina, Phys. Rev. Lett. 111, 241801 (2013). arXiv: 1307.5193 [hep-ph]

10. V. Branchina, E. Messina, A. Platania, JHEP 1409, 182 (2014). arXiv:1407.4112 [hep-ph]

11. V. Branchina, E. Messina, M. Sher, Phys. Rev. D 91, 013003 (2015). arXiv:1408.5302 [hep-ph]

12. V. Branchina, E. Messina, D. Zappala, EPL 116(2), 21001 (2016). arXiv:1601.06963 [hep-ph]

13. V. Branchina, E. Messina, EPL 117(6), 61002 (2017). arXiv:1507.08812 [hep-ph]

14. E. Bentivegna, V. Branchina, F. Contino, D. Zappal, JHEP 1712, 100 (2017). arXiv:1708.01138 [hep-ph]

15. N. Khan, S. Rakshit, Phys. Rev. D 90, 113008 (2014). arXiv:1407.6015 [hep-ph]

16. N. Khan, S. Rakshit, Phys. Rev. D 92, 055006 (2015). arXiv:1503.03085 [hep-ph]

17. A. Datta, N. Ganguly, N. Khan and S. Rakshit, arXiv: 1610.00648 [hep-ph]

18. L. Basso, O. Fischer, J.J. van Der Bij, Phys. Lett. B 730, 326 (2014). arXiv:1309.6086 [hep-ph]

19. O. Fischer, arXiv:1607.00282 [hep-ph]

20. T. Blank, W. Hollik, Nucl. Phys. B 514, 113 (1998). arXiv:hep-ph/9703392

21. J.R. Forshaw, A. Sabio Vera, B.E. White, JHEP 0306, 059 (2003). arXiv:hep-ph/0302256

22. M.C. Chen, S. Dawson, C.B. Jackson, Phys. Rev. D 78, 093001 (2008). arXiv:0809.4185 [hep-ph]

23. M.C. Chen, S. Dawson, T. Krupovnickas, Phys. Rev. D 74, 035001 (2006). arXiv:hep-ph/0604102

24. M.C. Chen, S. Dawson, T. Krupovnickas, Int. J. Mod. Phys. A 21, 4045 (2006). arXiv:hep-ph/0504286

25. P.H. Chankowski, S. Pokorski, J. Wagner, Eur. Phys. J. C 50, 919 (2007). arXiv:hep-ph/0605302

26. J.R. Forshaw, D.A. Ross, B.E. White, JHEP 0110, 007 (2001). arXiv:hep-ph/0107232

27. Z.U. Khandker, D. Li, W. Skiba, Phys. Rev. D 86, 015006 (2012). arXiv:1201.4383 [hep-ph]

28. P. Fileviez Perez, H.H. Patel, M.J. Ramsey-Musolf, K. Wang, Phys. Rev. D 79, 055024 (2009). arXiv:0811.3957 [hep-ph]

29. L. Wang, X.F. Han, JHEP 1403, 010 (2014). arXiv:1303.4490 [hep-ph]

30. N. Khan, B. Mukhopadhyaya, S. Rakshit, A. Shaw, arXiv:1608.05673 [hep-ph]

31. T. Araki, C.Q. Geng, K.I. Nagao, Phys. Rev. D 83, 075014 (2011). arXiv:1102.4906 [hep-ph]

32. S. Y. Ayazi, S. M. Firouzabadi, arXiv:1501.06176 [hep-ph]

33. S.Y. Ayazi, S.M. Firouzabadi, JCAP 1411, 005 (2014). arXiv: 1408.0654 [hep-ph]

34. F.X. Josse-Michaux, E. Molinaro, Phys. Rev. D 87, 036007 (2013). arXiv:1210.7202 [hep-ph]

35. W. B. Lu, P. H. Gu, JCAP 1605(05), 040 (2016). arXiv:1603.05074 [hep-ph]

36. O. Fischer, J.J. van der Bij, Mod. Phys. Lett. A 26, 2039 (2011)

37. O. Fischer, J.J. van der Bij, JCAP 1401, 032 (2014). arXiv: 1311.1077 [hep-ph]

38. K.A. Olive et al., Particle Data Group Collaboration. Chin. Phys. C 38, 090001 (2014)

39. B.W. Lee, C. Quigg, H.B. Thacker, Phys. Rev. Lett. 38, 883 (1977)

40. B.W. Lee, C. Quigg, H.B. Thacker, Phys. Rev. D 16, 1519 (1977)

41. Y.P. Yao, C.P. Yuan, Phys. Rev. D 38, 2237 (1988)

42. H.G.J. Veltman, Phys. Rev. D 41, 2294 (1990)
43. H.J. He et al., Phys. Rev. Lett. 69, 2619 (1992)

44. S. Kanemura et al., Phys. Lett. B 313, 155-160 (1993)

45. A. Arhrib, arXiv:hep-ph/0012353

46. M.E. Peskin, T. Takeuchi, Phys. Rev. D 46, 381 (1992)

47. M. Baak et al., Gfitter Group Collaboration. Eur. Phys. J. C 74, 3046 (2014). arXiv:1407.3792 [hep-ph]

48. G. Belanger, B. Dumont, U. Ellwanger, J.F. Gunion, S. Kraml, Phys. Rev. D 88, 075008 (2013)

49. A. Djouadi, Phys. Rept. 459, 1 (2008). arXiv:hep-ph/0503173

50. G. Aad et al., ATLAS Collaboration. Phys. Rev. D 90, 112015 (2014). arXiv:1408.7084 [hep-ex]

51. V. Khachatryan et al., CMS Collaboration. Eur. Phys. J. C 74, 3076 (2014). arXiv:1407.0558 [hep-ex]

52. K.G. Chetyrkin, M.F. Zoller, JHEP 1206, 033 (2012). arXiv:1205.2892 [hep-ph]

53. M. F. Zoller, arXiv:1209.5609 [hep-ph]

54. K.G. Chetyrkin, M.F. Zoller, JHEP 1304, 091 (2013). arXiv:1303.2890 [hep-ph]. [Erratum-ibid. 1309, 155 (2013)]

55. M. Zoller, PoS EPS-HEP2013, 322 (2014) arXiv:1311.5085 [hep$\mathrm{ph}]$

56. M. Cirelli, N. Fornengo, A. Strumia, Nucl. Phys. B 753, 178 (2006). arXiv:hep-ph/0512090

57. M. Cirelli, A. Strumia, New J. Phys. 11, 105005 (2009). arXiv:0903.3381 [hep-ph]

58. G. Belanger, B. Dumont, U. Ellwanger, J.F. Gunion, S. Kraml, Phys. Rev. D 88, 075008 (2013). arXiv:1306.2941 [hep-ph]

59. K. Griest, D. Seckel, Phys. Rev. D 43, 3191 (1991)

60. A. Alloul, N.D. Christensen, C. Degrande, C. Duhr, B. Fuks, Comput. Phys. Commun. 185, 2250 (2014). arXiv:1310.1921 [hep-ph]

61. G. Belanger, F. Boudjema, P. Brun, A. Pukhov, S. Rosier-Lees, P. Salati, A. Semenov, Comput. Phys. Commun. 182, 842 (2011). arXiv:1004.1092 [hep-ph]

62. G. Belanger, F. Boudjema, A. Pukhov, A. Semenov, Comput. Phys. Commun. 185, 960 (2014). arXiv:1305.0237 [hep-ph]

63. P.A.R. Ade et al. [Planck Collaboration]. arXiv:1303.5076 [astroph.CO]

64. E. Aprile et al., XENON100 Collaboration. Phys. Rev. Lett. 107, 131302 (2011). arXiv:1104.2549 [astro-ph.CO]

65. E. Aprile et al., XENON100 Collaboration. Phys. Rev. Lett. 109, 181301 (2012). arXiv:1207.5988 [astro-ph.CO]

66. D.S. Akerib et al., LUX Collaboration. Phys. Rev. Lett. 112, 091303 (2014). arXiv:1310.8214 [astro-ph.CO]

67. D.S. Akerib et al., Results from a search for dark matter in the complete LUX exposure. Phys. Rev. Lett. 118(2), 021303 (2017)

68. E. Aprile et al., XENON Collaboration. Phys. Rev. Lett. 119, 181301 (2017). arXiv:1705.06655 [astro-ph.CO]

69. J.A. Casas, J.R. Espinosa, M. Quiros, Phys. Lett. B 342, 171 (1995). arXiv:hep-ph/9409458

70. G. Altarelli, G. Isidori, Phys. Lett. B 337, 141 (1994)

71. J.A. Casas, J.R. Espinosa, M. Quiros, A. Riotto, Nucl. Phys. B 436, 3 (1995). arXiv:hep-ph/9407389 [Erratum-ibid. B 439, 466 (1995)]

72. J.A. Casas, J.R. Espinosa, M. Quiros, Phys. Lett. B 382, 374 (1996). arXiv:hep-ph/9603227

73. M. Quiros, arXiv:hep-ph/9703412

74. C. Ford, I. Jack, D.R.T. Jones, Nucl. Phys. B 387, 373 (1992). arXiv:hep-ph/0111190 [Erratum-ibid. B 504, 551 (1997)]

75. S.P. Martin, Phys. Rev. D 65, 116003 (2002). arXiv:hep-ph/0111209

76. A. Sirlin, R. Zucchini, Nucl. Phys. B 266, 389 (1986)

77. F. Bezrukov, M.Y. Kalmykov, B.A. Kniehl, M. Shaposhnikov, JHEP 1210, 140 (2012). arXiv:1205.2893 [hep-ph]

78. G. Isidori, G. Ridolfi, A. Strumia, Nucl. Phys. B 609, 387 (2001). arXiv:hep-ph/0104016

79. S. R. Coleman, Phys. Rev. D 15, 2929 (1977) [Erratum-ibid. D 16, 1248 (1977)] 
80. S.R. Coleman, F. De Luccia, Phys. Rev. D 21, 3305 (1980)

81. G. Isidori, V.S. Rychkov, A. Strumia, N. Tetradis, Phys. Rev. D 77, 025034 (2008). arXiv:0712.0242 [hep-ph]

82. L. Delle Rose, C. Marzo, A. Urbano, JHEP 1605, 050 (2016). arXiv:1507.06912 [hep-ph]

83. J.R. Espinosa, M. Quiros, Phys. Lett. B 353, 257 (1995). arXiv:hep-ph/9504241

84. F.L. Bezrukov, M. Shaposhnikov, Phys. Lett. B 659, 703 (2008). arXiv:0710.3755 [hep-th]

85. F. Bezrukov, D. Gorbunov, M. Shaposhnikov, JCAP 0906, 029 (2009). arXiv:0812.3622 [hep-ph]

86. F.L. Bezrukov, A. Magnin, M. Shaposhnikov, Phys. Lett. B 675, 88 (2009). arXiv:0812.4950 [hep-ph]

87. F. Bezrukov, M. Shaposhnikov, JHEP 0907, 089 (2009). arXiv:0904.1537 [hep-ph]

88. A.O. Barvinsky, A.Y. Kamenshchik, A.A. Starobinsky, JCAP 0811, 021 (2008). arXiv:0809.2104 [hep-ph]

89. A.O. Barvinsky, A.Y. Kamenshchik, C. Kiefer, A.A. Starobinsky, C. Steinwachs, JCAP 0912, 003 (2009). arXiv:0904.1698 [hep$\mathrm{ph}]$

90. A. De Simone, M.P. Hertzberg, F. Wilczek, Phys. Lett. B 678, 1 (2009). arXiv:0812.4946 [hep-ph]

91. J. Garcia-Bellido, D.G. Figueroa, J. Rubio, Phys. Rev. D 79, 063531 (2009). arXiv:0812.4624 [hep-ph]
92. S.C. Park, S. Yamaguchi, JCAP 0808, 009 (2008). arXiv:0801.1722 [hep-ph]

93. R.N. Lerner, J. McDonald, Phys. Rev. D 80, 123507 (2009). arXiv:0909.0520 [hep-ph]

94. O. Lebedev, H.M. Lee, Eur. Phys. J. C 71, 1821 (2011). arXiv:1105.2284 [hep-ph]

95. G.K. Chakravarty, S. Mohanty, Phys. Lett. B 746, 242 (2015). arXiv:1405.1321 [hep-ph]

96. G.K. Chakravarty, G. Gupta, G. Lambiase, S. Mohanty, Phys. Lett. B 760, 263 (2016). arXiv:1604.02556 [hep-ph]

97. G.K. Chakravarty, U.K. Dey, G. Lambiase, S. Mohanty, Phys. Lett. B 763, 501 (2016). arXiv:1607.06904 [hep-ph]

98. J. Ellis, arXiv:1702.05436 [hep-ph]

99. F. Kahlhoefer, J. McDonald, JCAP 1511(11), 015 (2015). arXiv:1507.03600 [astro-ph.CO]

100. P.A.R. Ade et al., Planck Collaboration. Astron. Astrophys. 594, A13 (2016). arXiv:1502.01589 [astro-ph.CO]

101. P.A.R. Ade et al., Planck collaboration. Astron. Astrophys. 594, A20 (2016). arXiv:1502.02114 [astro-ph.CO]

102. F. Staub, Comput. Phys. Commun. 185, 1773 (2014). arXiv: 1309.7223 [hep-ph]

103. F. Staub, arXiv: 1503.04200 [hep-ph]

104. K. Kannike, Eur. Phys. J. C 72, 2093 (2012). arXiv:1205.3781 [hep-ph] 